\title{
Linear Positivity and Virtual Probability
}

\author{
James B. Hartle* \\ Department of Physics \\ University of California, Santa Barbara, CA 93106-9530
}

(Dated: October 30, 2018)

\begin{abstract}
We investigate the quantum theory of closed systems based on the linear positivity decoherence condition of Goldstein and Page. The objective of any quantum theory of a closed system, most generally the universe, is the prediction of probabilities for the individual members of sets of alternative coarse-grained histories of the system. Quantum interference between members of a set of alternative histories is an obstacle to assigning probabilities that are consistent with the rules of probability theory. A quantum theory of closed systems therefore requires two elements; 1) a condition specifying which sets of histories may be assigned probabilities, and 2) a rule for those probabilities.

The linear positivity condition of Goldstein and Page is the weakest of the general conditions proposed so far. Its general properties relating to exact probability sum rules, time-neutrality, and conservation laws are explored. Its inconsistency with the usual notion of independent subsystems in quantum mechanics is reviewed. Its relation to the stronger condition of medium decoherence necessary for classicality is discussed. The linear positivity of histories in a number of simple model systems is investigated with the aim of exhibiting linearly positive sets of histories that are not decoherent. The utility of extending the notion of probability to include values outside the range of 0 to 1 is described. Alternatives with such virtual probabilities cannot be measured or recorded, but can be used in the intermediate steps of calculations of real probabilities. Extended probabilities give a simple and general way of formulating quantum theory.

The various decoherence conditions are compared in terms of their utility for characterizing classicality and the role they might play in further generalizations of quantum mechanics.
\end{abstract}

PACS numbers: PACS 03.65.Yz, 03.65.Ta, 98.80Qc

\section{INTRODUCTION}

The familiar "Copenhagen" quantum mechanics of measured subsystems must be generalized to apply to closed systems such as the universe as a whole. That is because there is nothing outside a closed system to measure it. Rather measurement situations occur within a closed system containing both the subsystem observed, and the observers, apparatus, etc. observing it. Consistent (or decoherent) histories quantum theory provides such a generalization. ${ }^{1}$ This paper is concerned with the nature of the decoherence conditions which are central to these formulations of quantum theory.

The most general predictions of a quantum theory of a closed system are the probabilities of coarse-grained alternative histories from the system's initial quantum state and Hamiltonian. The probabilities for alternative orbits of the Earth around the Sun are examples. An orbit might be described by a series of center-of-mass position intervals at a sequence of times. That description is coarse-grained because it is specified only by center of mass position and not all possible variables, because those positions are specified only at some times and not at all times, and because they are specified only within intervals and not to arbitrary accuracy. Such coarse-graining is generally necessary to have probabilities at all.

Copenhagen quantum mechanics predicts the probabilities

\footnotetext{
*Electronic address: hartle@physics.ucsb.edu

${ }^{1}$ See e.g. [1, 2, 3] for extended descriptions from various points of view.
}

of histories of measurement outcomes, and the quantum mechanics of closed systems predicts these also. However, these generalizations of Copenhagen quantum mechanics also predict probabilities for histories of subsystems even when these are not receiving attention from observers. The history of the Earth's motion in the first few billion years of its existence is an example.

Not every set of histories that can be described can be assigned probabilities that are consistent with the rules of probability theory, in particular, with the rule that the probability of two exclusive alternatives is the sum of the probabilities of each. Quantum interference is the obstacle. In usual quantum mechanics, for instance, probabilities are the squares of amplitudes and the square of a sum is not generally the sum of the squares. A quantum theory of closed systems can therefore be based on two elements: 1) An expression for computing probabilities and 2) a consistency condition that specifies which sets of alternative histories can be consistently assigned probabilities.

A variety of conditions enforcing consistency have been proposed and studied (See, e.g. [4] for a list). This paper is concerned with the weakest of these - linear positivity introduced in a seminal paper by Goldstein and Page [5]. Linear positivity will be described in Sections II and III in detail, but to introduce the idea let us first recall the medium decoherence condition.

Individual histories in an exhaustive set of exclusive alternative histories are represented by operators $C_{\alpha}$ called class operators where $\alpha$ labels the histories in the set. For instance, the history in which the orbit of the Earth lies in a sequence of ranges of position at a series of times would be represented 
by a $C_{\alpha}$ which is the time-ordered product of projections onto these ranges at the different times. The class operators for an exhaustive set of histories satisfy

$$
\sum_{\alpha} C_{\alpha}=I, \quad \text { (exhaustive). }
$$

If $|\Psi\rangle$ is the state of the closed system, the class operators can be used to construct branch state vectors $\left|\Psi_{\alpha}\right\rangle$ for each history according to

$$
\left|\Psi_{\alpha}\right\rangle \equiv C_{\alpha}|\Psi\rangle .
$$

The probabilities of the individual histories are given by

$$
p(\alpha)=\|\left|\Psi_{\alpha}\right\rangle\left\|^{2}=\right\| C_{\alpha}|\Psi\rangle \|^{2} .
$$

Medium decoherence is the condition that the branches be orthogonal

$$
\begin{aligned}
\left\langle\Psi_{\alpha} \mid \Psi_{\alpha^{\prime}}\right\rangle= & \left\langle\Psi\left|C_{\alpha}^{\dagger} C_{\alpha^{\prime}}\right| \Psi\right\rangle=0 \quad \text { all } \quad \alpha \neq \alpha^{\prime}, \\
& \text { (Medium Decoherence) }
\end{aligned}
$$

Medium decoherence is the precise representation of the absence of interference between histories. It is sufficient for the consistency of the probabilities 1.3. However, it is not a necessary condition; weaker conditions are possible.

As a consequence of (1.1), 1.3), and (1.4), the probabilities of a medium decoherent set of histories can be re-expressed as

$$
p(\alpha) \equiv\left\langle\Psi_{\alpha} \mid \Psi_{\alpha}\right\rangle=\sum_{\alpha^{\prime}}\left\langle\Psi_{\alpha^{\prime}} \mid \Psi_{\alpha}\right\rangle=\left\langle\Psi \mid \Psi_{\alpha}\right\rangle=\left\langle\Psi\left|C_{\alpha}\right| \Psi\right\rangle .
$$

The numbers $\left\langle\Psi\left|C_{\alpha}\right| \Psi\right\rangle$ are real and positive as a consequence of medium decoherence (1.4). Goldstein and Page [5] proposed the weaker decoherence condition

$$
\operatorname{Re}\left\langle\Psi\left|C_{\alpha}\right| \Psi\right\rangle \geq 0, \quad \text { all } \alpha, \quad \text { (Linear Positivity), }
$$

replacing (1.4), and a new assignment of probability

$$
p(\alpha) \equiv \operatorname{Re}\left\langle\Psi\left|C_{\alpha}\right| \Psi\right\rangle,
$$

replacing (1.3). This is linear positivity. "Linear" refers to the linearity of the expression for probabilities 1.7) in the $C_{\alpha}$. "Positivity" characterizes the condition (1.6). As we will review in Section III, the linear positivity condition (1.6) is sufficient for the probabilities defined by (1.7) to be consistent.

The reality and positivity of the $\left\langle\Psi\left|C_{\alpha}\right| \Psi\right\rangle$ that follow from (1.5) show that medium decoherence implies linear positivity but not the other way around. Linear positivity thus extends the sets of histories that can be assigned probabilities beyond those which are medium decoherent. That extension is not necessary to describe the outcomes of measurements. Sets of histories describing measurements are medium decoherent, at least to an excellent approximation, as we discuss in Section III.A.

Linear positivity is the weakest of the general conditions for consistent probabilities of histories known to the author at the time of writing. However mere consistency with probability sum rules may not be the only requirement reasonably imposed on quantum mechanical probabilities. For example, Diosi [7] pointed out that linear positivity is inconsistent with the usual notion of statistically independent subsystems in quantum mechanics. The Hilbert space of a closed system of $N$, non-interacting subsystems is the tensor product of the Hilbert spaces for the individual subsystems. States representing statistically independent subsystems have the product form

$$
|\Psi\rangle=\left|\Psi_{1}\right\rangle \otimes \cdots \otimes\left|\Psi_{N}\right\rangle
$$

where the $\left|\Psi_{i}\right\rangle$ are the states of the individual subsystems. The probability for the ensemble that the independent subsystems have a sequence of properties should be the product of the probabilities of the properties in the individual subsystems. While medium decoherence guarantees this property, linear positivity does not, as Diosi showed and as we review in Section III.D. For the author this is reason enough to reject linear positivity as a basis for the quantum mechanics of closed systems that contain isolated subsystems.

However, usual quantum mechanics does not need to be generalized for quantum cosmology only because the universe is a closed system. A generalization is needed to accommodate quantum gravity. That is because even the quantum framework alluded to above, and described more carefully in the next section, relies on a fixed background spacetime geometry for example to define the notion of time employed. But in a quantum theory of gravity spacetime geometry is a quantum dynamical variable, generally fluctuating and without definite value. The notion of an exactly isolated subsystem is problematical in a closed universe because the gravitational interaction cannot be screened. It is therefore just possible that linear positivity could be useful could be useful for a generalization of quantum theory incorporating quantum gravity. It is in this spirit that we explore linear positivity in this paper, although no gravitational issues will be addressed explicitly.

This paper explores linear positivity in a number of different directions. General properties such as exact probability sum rules, time neutrality, conservation laws, the relation to medium decoherence, and the inconsistency with the usual notion of statistically independent subsystems are discussed in Section III. Section IV exhibits examples of linear positivity in a variety of simple, non-relativistic quantum mechanical systems - the two-slit experiment, the three-box model, a spin-1/2 system, a free particle, and alternatives extended over time. The aim is to begin a kind of phenomenology of linear positivity. In general, a much wider range of histories of this simple kind are found to be linearly positive than are decoherent. Section V describes the utility of the numbers defined by (1.7) even when they are not positive or less than unity. We call these virtual probabilities. Alternatives with virtual probabilities can neither be measured nor recorded but can be used as intermediate steps in calculations of real probabilities. We discuss the advantages and disadvantages of formulating quantum theory in terms of both in Section VI. 


\section{QUANTUM MECHANICS OF CLOSED SYSTEMS}

This section very briefly reviews the essentials of the quantum mechanics of closed systems [6] especially to fix the notation to be used in the rest of the paper.

We consider a closed system, most generally the universe as a whole, in the approximation that gross quantum fluctuations in the geometry of spacetime (i.e. quantum gravity) can be neglected. The closed system can then be thought of as a very large box of particles and fields containing both observers and observed (if any). Fixed background spacetime means that a notion of time is well defined and the usual apparatus of Hilbert space, states, and operators can be employed in a quantum description of the box. The quantum state of this closed system $|\Psi\rangle$ (represented as a wave function of the universe) and the Hamiltonian $H$ describing its quantum evolution are assumed to be given and fixed.

As mentioned in the Introduction, the most general objective of quantum theory for a closed system is the prediction of probabilities for the individual members of sets of coarsegrained alternative histories. The simplest sets of alternative histories are specified by alternatives at one moment of time. These can always be reduced to a set of yes/no alternatives represented by an exhaustive set of orthogonal projection operators $\left\{P_{\alpha}(t)\right\} \alpha=1,2, \cdots$ in the Heisenberg picture. These projections satisfy

$$
\sum_{\alpha} P_{\alpha}(t)=I, \quad \text { and } \quad P_{\alpha}(t) P_{\beta}(t)=\delta_{\alpha \beta} P_{\alpha}(t)
$$

showing that they represent an exhaustive set of exclusive alternatives. The operators $P_{\alpha}(t)$ change with time according to the Heisenberg equation of motion defined by $H$. The state $|\Psi\rangle$ is unchanging in the Heisenberg picture.

A set of alternative histories may be specified by giving sets of alternatives $\left\{P_{\alpha_{1}}^{1}\left(t_{1}\right)\right\},\left\{P_{\alpha_{2}}^{2}\left(t_{2}\right)\right\}, \cdots,\left\{P_{\alpha_{n}}^{n}\left(t_{n}\right)\right\}$ at a series of times $t_{1}<t_{2}, \cdots,<t_{n}$. The sets at distinct times can differ and are distinguished by the superscript on the $P$ 's. The subscript distinguishes the alternatives within the set. An individual history in this set is represented by a particular sequence of alternatives $\left(\alpha_{1}, \cdots, \alpha_{n}\right) \equiv \alpha$ and is represented by the corresponding chain of projections

$$
C_{\alpha}=P_{\alpha_{n}}^{n}\left(t_{n}\right) \cdots P_{\alpha_{1}}^{1}\left(t_{1}\right)
$$

Such a set of histories is coarse grained because alternatives are specified at some times and not at every time and because the specified alternatives are projections on subspaces with dimension larger than one and not projections on a complete set of operators.

The operations of coarse and fine graining relate different sets of histories. A set of histories $\left\{C_{\alpha}\right\}$ may be coarse grained by partitioning it into an exhaustive set of exclusive classes $\bar{c}_{\bar{\alpha}}, \bar{\alpha}=1,2, \cdots$. Each class consists of some number of histories in the finer-grained set and every finer-grained history lies in some class. Fine graining is the inverse operation of dividing the histories up into mutually exclusive smaller classes. The class operators $\left\{\bar{C}_{\bar{\alpha}}\right\}$ for the histories in a coarse graining of a set whose class operators are $\left\{C_{\alpha}\right\}$ are related by summa- tion

$$
\bar{C}_{\bar{\alpha}}=\sum_{\alpha \in \bar{\alpha}} C_{\alpha}
$$

where the sum defining the coarse-grained history $\bar{C}_{\bar{\alpha}}$ is over all the finer-grained histories contained within it.

Probabilities for a set of histories are a set of numbers $p(\alpha)$ lying between 0 and 1

$$
0 \leq p(\alpha) \leq 1
$$

that satisfy the basic probability sum rules relating finer- to coarser-grained descriptions: The probability of a coarsegrained set is the sum of the probabilities of its finer-grained members. The probabilities of the coarser-grained descriptions are then consistent with those of the finer-grained ones. Applied to histories for a coarse graining of the form (2.3) this is the requirement

$$
\bar{p}(\bar{\alpha})=\sum_{\alpha \in \bar{\alpha}} p(\alpha)
$$

where the $\bar{p}(\bar{\alpha})$ are the probabilities of the $\bar{C}_{\bar{\alpha}}$, and $p(\alpha)$ are the probabilities of the $C_{\alpha}$. The two conditions 2.4 are the mathematical requirements for probabilities.

The Introduction described how quantum interference is an obstacle to consistency in the sense of 2.4b and the need for a decoherence condition to restrict the sets to ones where it is satisfied. The next Section describes the Goldstein and Page [5] linear positivity condition and some of its general properties.

\section{LINEAR POSITIVITY}

The linear positivity condition concerns the numbers

$$
p(\alpha) \equiv \operatorname{Re}\left\langle\Psi\left|C_{\alpha}\right| \Psi\right\rangle=\frac{1}{2}\left\langle\Psi\left|\left(C_{\alpha}+C_{\alpha}^{\dagger}\right)\right| \Psi\right\rangle
$$

which can be calculated for any set of histories $\left\{C_{\alpha}\right\}$. It's convenient to call these candidate probabilities and use the notation $p(\alpha)$ for candidate probabilities whether or not their range is between 0 and 1 . Because of 2.3, they automatically and exactly satisfy the necessary sum rules (2.4b) for the probabilities of a coarse graining of the set. However, candidate probabilities do not necessarily lie between 0 and 1 as (2.4a) requires. Indeed, even for histories with just two times, with class operators of the form $C_{\alpha_{2} \alpha_{1}}=P_{\alpha_{2}}^{2}\left(t_{2}\right) P_{\alpha_{1}}^{1}\left(t_{1}\right)$, there is some state in which one of the histories has a negative $p(\alpha)$. That is because the Hermitian product of two non-commuting projections always has at least one negative eigenvalue (Appendix A).

Homogeneous histories whose class operators are chains of projections as in 2.2 have candidate probabilities that are less than unity. That is because the action of a non-trivial projection on a state always reduces its norm.

$$
\operatorname{Re}\left\langle\Psi\left|P_{\alpha_{n}}^{n}\left(t_{n}\right) \cdots P_{\alpha_{1}}^{1}\left(t_{1}\right)\right| \Psi\right\rangle \leq 1
$$


There is no guarantee that a coarse graining of a set of homogeneous histories that does not itself consist of homogeneous histories will have $p(\alpha)<1$. Indeed, for any set of homogeneous histories with at least one negative $p(\alpha)$, there is always a coarse graining in which at least one history has $\bar{p}(\bar{\alpha}) \geq 1$. However, if the $p(\alpha)$ are all positive or zero then they are also less than 1 because they must sum to 1 as a consequence of 1.1. Genuine probabilities are therefore guaranteed by the linear positivity condition (1.6).

Section IV will illustrate the linear positivity condition for simple systems. But first we consider some of its general properties and in particular its relation to medium decoherence.

\section{A. Connection with Medium Decoherence}

A set of histories medium decoheres when

$$
\left\langle\Psi\left|C_{\alpha}^{\dagger} C_{\beta}\right| \Psi\right\rangle=0, \quad \alpha \neq \beta \quad \text { (Medium Decoherence). }
$$

The probabilities of the individual histories in a medium decoherent set is

$$
p^{\mathrm{MD}}(\alpha)=\left\langle\Psi\left|C_{\alpha}^{\dagger} C_{\alpha}\right| \Psi\right\rangle=\| C_{\alpha}|\Psi\rangle \|^{2}
$$

As here, we use superscripts MD and LP where necessary to distinguish medium decoherent and linear positivity probabilities.

The identity $\sum_{\beta} C_{\beta}=I$ [cf. 1.1] $]$ can be used to derive the following relation between the $p^{\mathrm{LP}}(\alpha)$ given by 3.1 and the $p^{\mathrm{MD}}(\alpha)$ given by 3.4

$$
p^{\mathrm{LP}}(\alpha)=p^{\mathrm{MD}}(\alpha)+\sum_{\beta \neq \alpha} \operatorname{Re}\left\langle\Psi\left|C_{\beta}^{\dagger} C_{\alpha}\right| \Psi\right\rangle
$$

Evidently, the two notions of probability coincide if the set is exactly medium decoherent and the last term vanishes [ $c f$. (3.3)]. Exact medium decoherence implies linear positivity.

However, many realistic coarse grainings are unlikely to medium decohere exactly, but only to an excellent approximation. For example, a branch state vector defining coarsegrained history of the motion of the Earth to an accuracy of $1 \mathrm{~cm}$ every second is not exactly orthogonal to a branch state vector of a distinct history. Rather it is approximately orthogonal to an accuracy far beyond that to which the resulting probabilities can be checked or the physical situation modeled. Approximate medium decoherence does not necessarily imply linear positivity because the second term in 3.5 may have any sign. If the $p^{\mathrm{MD}}(\alpha)$ are very small, $p^{\mathrm{LP}}(\alpha)$ could be negative. (But then, approximate medium decoherence would imply approximate linear positivity.)

Medium decoherence can be alternatively characterized by the existence of records of the histories. There are records of a set of histories if there is an exhaustive set of orthogonal commuting projections $\left\{R_{\beta}\right\}$ which are correlated with the histories $\left\{C_{\alpha}\right\}$ in the following strong sense

$$
R_{\beta} C_{\alpha}|\Psi\rangle=\delta_{\beta \alpha} C_{\alpha}|\Psi\rangle
$$

Evidently 3.6 implies the medium decoherence condition (3.3), because of the orthogonality of the R's. Conversely, if the branches $\left|\Psi_{\alpha}\right\rangle=C_{\alpha}|\Psi\rangle$ are orthogonal, there are generally many different sets of projections $\left\{R_{\alpha}\right\}$ for which (3.6) is satisfied - most simply $R_{\alpha}=\left|\Psi_{\alpha}\right\rangle\left\langle\Psi_{\alpha}\right|$. The $\left\{R_{\alpha}\right\}$ are records only in the abstract sense specified of (3.6) and need not correspond to anything like everyday records such as history books. The creation and persistence of records is an important, indeed the defining, feature of many realistic mechanisms of decoherence.

\section{B. Exact Probabilities}

As stressed by Goldstein and Page, and as mentioned above, the probabilities of a linearly positive set of histories satisfy the defining probability sum rules $2.4 \mathrm{~b}$ exactly. Probabilities need to be defined by physical theory only up to the accuracy they are used. For example, we consider a prediction of the frequency of outcomes of repeated experiments to be securely tested, not if the probability of a significant fluctuation in the frequency of expected outcomes is exactly zero, but rather if it is sufficiently small. Two theories that predict probabilities whose difference is well below the standard with which they are used are equivalent in predictive power. Therein lies the possibility of utilizing the probabilities of realistic approximately medium decoherent sets of histories that obey the sum rules 2.4b only to an approximation secure beyond all test. However, the conceptual situation is considerably simplified if the predicted probabilities exactly obey the sum rules as they do in the linearly positive case. We will return to the relation between these two cases in Section VI.

\section{Exact Conservation Laws}

Exact probability sum rules ensure that quantities that commute with the Hamiltonian are conserved with probability 1. The argument is essentially the same as that in [8] but we provide a sketch of it here.

Consider a quantity $A$ like total electric charge or total energy that commutes with the Hamiltonian $H$. Let $P_{j}^{\mathrm{A}}(t)$ denote a set of projections onto disjoint ranges $\Delta_{j}, j=1,2,3, \ldots$ of $A$ at time $t$. Orthogonality of these projections implies

$$
P_{j^{\prime}}^{\mathrm{A}}\left(t^{\prime}\right) P_{j}^{\mathrm{A}}(t)=0, \quad j \neq j^{\prime}
$$

because $A$ commutes with $H$. Now consider a set of histories of the form

$$
C_{\alpha}=P_{j^{\prime}}^{\mathrm{A}}\left(t^{\prime}\right) C_{\beta}^{b} P_{j}^{\mathrm{A}}(t)
$$

where $C_{\beta}^{b}$ is a chain of projections at times in between $t$ and $t^{\prime}$ onto ranges of quantities not necessarily commuting with $A$. Is the value of the conserved quantity $A$ at $t^{\prime}$ exactly correlated with the value at $t$ despite the intervening projections on quantities not necessarily commuting with $H$ ? Linear positivity 
ensures that it is. Let $p\left(j^{\prime}, \beta, j\right)$ denote the candidate probabilities of the histories (3.8) computed according to (3.1). The sum rules $2.4 \mathrm{~b}$ ensure

$$
\sum_{\beta} p\left(j^{\prime}, \beta, j\right)=p\left(j^{\prime}, j\right)=\delta_{j^{\prime} j} p(j)
$$

from (3.7). If the $p$ 's are all positive as linear positivity requires, then (3.9) implies

$$
p\left(j^{\prime}, \beta, j\right)=0, \quad j^{\prime} \neq j
$$

which is exact conservation of the quantity $A$.

\section{Statistical Probabilities}

The probabilities under discussion are probabilities for particular histories of a single closed system ${ }^{2}$. When the closed system consists of an ensemble of identical subsystems, the probabilities of the closed system can be used to discuss the statistics of the ensemble when the number of its members is large. For example, with high probability, the frequency of a particular history in the ensemble should equal its probability in an individual subsystem. That is a standard result in usual quantum theory [10] in accord with the law of large numbers. However, as observed by Diosi [7], linear positivity is generally inconsistent with the usual notion of statistical independence in quantum theory on which such demonstrations are based. We offer a brief review of his result.

In familiar quantum theory, an ensemble of $N$ identical, non-interacting subsystem is represented by a state

$$
|\Psi\rangle=|\Psi\rangle \otimes \cdots \otimes|\Psi\rangle
$$

on the tensor product of $N$ copies of the Hilbert space $\mathcal{H}$ of the individual subsystems. A closed system with a state 3.11 can be thought of as an approximate model for a realistic ensemble of identical subsystems. Alternatively, even when $|\Psi\rangle$ refers to the universe, 3.11 can be thought of as describing a fictitious ensemble of universes whose frequencies can be expected to coincide with the probabilities of the individual members in the limit of large $N$.

Let $\left\{C_{\alpha}\right\}$ denote the class operators acting on $\mathcal{H}$ for a set of alternative, coarse-grained histories of a subsystem. The class operator for $N$ different histories $\alpha^{1}, \cdots, \alpha^{N}$ in the ensemble is

$$
\mathbf{C}_{\alpha^{1 \cdots \alpha^{N}}}=C_{\alpha^{1}} \otimes \cdots \otimes C_{\alpha^{N}}
$$

[We employ a superscript to distinguish histories $\alpha^{1}, \cdots, \alpha^{N}$ of different subsystems each of which may consist of a sequence of alternatives e.g. $\quad \alpha^{1}=\left(\alpha_{1}^{1}, \cdots, \alpha_{n}^{1}\right) ; \alpha^{2}=$ $\left(\alpha_{1}^{2}, \cdots, \alpha_{n}^{2}\right)$; etc.] Suppose that the set of histories $\left\{C_{\alpha}\right\}$ of

\footnotetext{
${ }^{2}$ See e.g. [9] Section II.2 for a discussion of how these probabilities are used and interpreted.
}

a subsystem is medium decoherent (3.3) so that in particular [cf. (3.4)]

$$
p^{\mathrm{MD}}(\alpha)=\| C_{\alpha}|\Psi\rangle \|^{2} .
$$

Then for the ensemble, evidently

$$
\begin{aligned}
\mathbf{p}^{\mathrm{MD}}\left(\alpha^{1}, \cdots, \alpha^{N}\right) & =\| \mathbf{C}_{\alpha^{1} \cdots \alpha^{N}}|\Psi\rangle \|^{2}, \\
& =\| C_{\alpha^{1}}|\Psi\rangle\left\|^{2} \cdots\right\| C_{\alpha^{N}}|\Psi\rangle \|^{2}, \\
& =p^{\mathrm{MD}}\left(\alpha^{1}\right) \cdots p^{\mathrm{MD}}\left(\alpha^{N}\right) .
\end{aligned}
$$

The subsystems are therefore statistically independent. This is the central fact in deriving the result that for a very large ensemble the frequency of an individual history $C_{\alpha}$ approaches its probability $p^{\mathrm{MD}}(\alpha)$ in an individual subsystem.

However, linear positivity does not yield the same notion of statistical independence. That is because [cf. 3.1] ]

$$
\begin{aligned}
\mathbf{p}^{\mathrm{LP}}\left(\alpha^{1}, \cdots, \alpha^{N}\right) & =\operatorname{Re}\left[\left\langle\Psi\left|\mathbf{C}_{\alpha^{1} \ldots \alpha^{N}}\right| \Psi\right\rangle\right], \\
& =\operatorname{Re}\left[\left\langle\Psi\left|C_{\alpha^{1}}\right| \Psi\right\rangle \cdots\left\langle\Psi\left|C_{\alpha^{N}}\right| \Psi\right\rangle\right]
\end{aligned}
$$

But the real part of a product is not generally the product of the real parts unless the individual factors are all real. Thus

$$
\mathbf{p}^{\mathrm{LP}}\left(\alpha^{1}, \cdots, \alpha^{N}\right) \neq p^{\mathrm{LP}}\left(\alpha^{1}\right) \cdots p^{\mathrm{LP}}\left(\alpha^{N}\right) .
$$

The absence of general equality in (3.16) does not mean that linear positivity incorrectly predicts the frequencies of the histories of the outcomes of measurements carried out on ensembles of idential subsystems of the universe. Exactly measured or exactly recorded alternatives are exactly medium decoherent and then (3.16) becomes an equality [cf.[3.14)]. However, for many realistic sets of alternative histories, including those describing the quasiclassical realm of everyday experience, medium decoherence and records are correlated with the alternatives they record only to excellent approximations. In such cases the (3.16) will only be an equality to a related approximation.

There is a second reason that linear positivity does not incorrectly predict the frequencies of individual histories in an ensemble of identical subsystems. Linear positivity fails for ensembles with a sufficiently large number of identical subsystems even if it is satisfied for the individual members unless the $\left\langle\Psi\left|C_{\alpha}\right| \Psi\right\rangle$ are real and (3.16) satisfied as consequence. To see this, consider a set of histories of the subsystem with just two members: $C_{1}$ and $C_{2}=I-C_{1}$ (so that 1.1 is satisfied.) Write the expected value of $C_{1}$ in terms of its magnitude and phase

$$
\left\langle\Psi\left|C_{1}\right| \Psi\right\rangle \equiv A e^{i \phi}
$$

with $A$ real and positive and $\phi$ real. Linear positivity requires $0<\phi<\pi$.

A given history of an ensemble of $N$ of these subsystems will have some number $n_{C}\left(0 \leq n_{C} \leq N\right)$ of histories $C_{1}$ and $N-n_{C}$ histories $C_{2}=I-C_{1}$. The candidate probabilities $p\left(n_{C}\right)$ for histories of the ensemble with $n_{C}$ individual histories $C_{1}$ are

$$
p\left(n_{C}\right)=\operatorname{Re}\left[\left(A e^{i \phi}\right)^{n_{C}}\left(1-A e^{i \phi}\right)^{N-n_{C}}\right] .
$$


For the histories of the ensemble to be linearly positive these candidate probabilities must be positive for all $n_{C}$ between 0 and $N$. However, unless $\phi$ is identically zero, there is some large $N$ for which (3.18) will not be positive for some $n_{C}$. Linear positivity thus fails for sufficiently large ensembles unless the $\left\langle\Psi\left|C_{\alpha}\right| \Psi\right\rangle$ are real . The failure means probabilities cannot be assigned to individual histories of the ensemble much less to values of the frequency of any history of its individual subsystems ${ }^{3}$.

A decoherence condition that would ensure equality in (3.16) and consistency with the usual notion of independent subsystems is

$$
\begin{aligned}
& \operatorname{Im}\left[\left\langle\Psi\left|C_{\alpha}\right| \Psi\right\rangle\right]=0, \quad \text { all } \alpha, \\
& \operatorname{Re}\left\langle\Psi\left|C_{\alpha}\right| \Psi\right\rangle \geq 0, \quad \text { all } \alpha, \\
& \text { (Real Linear Positivity) }
\end{aligned}
$$

This is linear positivity 1.6 augmented by the reality requirement 3.19a. At the risk of introducing more confusing terminology this might be called real linear positivity. This is weaker than medium decoherence because (1.5) and (1.2) show that it is equivalent to

$$
\operatorname{Im} \sum_{\beta}\left[\left\langle\Psi\left|C_{\beta}^{\dagger} C_{\alpha}\right| \Psi\right\rangle\right]=0 .
$$

Only the vanishing of the imaginary part of this sum is required for equality in (3.16), but medium decoherence ensures that each term with $\alpha \neq \beta$ vanishes separately. But, as with medium decoherence, (3.20) is likely to be satisfied only to an excellent approximation for realistic coarse grainings defining the quasiclassical realm.

The conflict between the usual notion of independent subsystems and linear positive probabilities is a serious obstacle to their interpretation when they are not associated with medium decoherent sets of histories. For example, we cannot understand them as frequencies in an imaginary ensemble identical copies of the closed system as described above. Indeed, it would not be unreasonable to impose 3.14 as a condition for a construction of probabilities in quantum mechanics thereby ruling out linear positivity. However, all this does not mean linear positive probabilities cannot be useful as we discuss in Section VI.

\section{E. Time Symmetry and Asymmetry}

Quantum theory is usually formulated with an arrow of time $^{4}$. Nowhere is that seen more clearly than in the standard formula [cf. 3.4] ] for the probability $p^{\mathrm{MD}}\left(\alpha_{n}, \cdots, \alpha_{1}\right)$ of a history of alternatives $\alpha_{1}, \cdots, \alpha_{n}$ at a sequence of times $t_{1}<t_{2}<\cdots<t_{n}$.

$$
p^{\mathrm{MD}}\left(\alpha_{n}, \cdots, \alpha_{1}\right)=\| P_{\alpha_{n}}^{n}\left(t_{n}\right) \cdots P_{\alpha_{1}}^{1}\left(t_{1}\right)|\Psi\rangle \|^{2} .
$$

\footnotetext{
${ }^{3}$ The author thanks S. Goldstein for stressing this point.

${ }^{4}$ See, e.g. [11] for a fuller discussion in the notation of this paper.
}

The state occurs at one end of the histories; there is nothing at the other end. That is the quantum mechanical arrow of time. It is a convention that $|\Psi\rangle$ enters as an initial condition earlier than all the alternatives. Field theory is CPT invariant and utilizing a CPT transformation the time order of the operators could be inverted so that $|\Psi\rangle$ is a final condition. However, no CPT transformation can alter the asymmetry between initial and final conditions in 3.21.

As discussed by Goldstein and Page, the linear positivity formula for probabilities [cf. (3.1] ] is time-neutral, viz.

$$
\begin{aligned}
p^{\mathrm{LP}}\left(\alpha_{n}, \cdots, \alpha_{1}\right) & =\operatorname{Re}\left\langle\Psi\left|P_{\alpha_{n}}^{n}\left(t_{n}\right) \cdots P_{\alpha_{1}}^{1}\left(t_{1}\right)\right| \Psi\right\rangle \\
& =\operatorname{Re}\left\langle\Psi\left|P_{\alpha_{1}}^{1}\left(t_{1}\right) \cdots P_{\alpha_{n}}^{n}\left(t_{n}\right)\right| \Psi\right\rangle .
\end{aligned}
$$

The ends of histories are treated symmetrically. ${ }^{5}$.

If the more general notion of linear positivity is fundamental in quantum theory, what is the origin of the time asymmetry displayed by the medium decoherence expression (3.21)? The answer is that the approximate equality of $p^{\mathrm{LP}}\left(\alpha_{n}, \cdots, \alpha_{1}\right)$ and $p^{\mathrm{MD}}\left(\alpha_{n}, \cdots, \alpha_{1}\right)$ can be expected to hold only for one ordering of the projections in 3.21). Put differently, the second term in 3.5 is not time-neutral. It will generally be small only for one-time ordering of the projections in $C_{\alpha}$ and not both. Only in trivial cases, e.g. projections on conserved quantities will the probabilities of an ordering and the inverse ordering agree.

Records of history connect the quantum mechanical arrow of time in 3.21) with the second law of thermodynamics for sets of histories constituting the quasiclassical realm of everyday experience. The histories of the quasiclassical realm [12, 13, 14, 15] consist of projections on ranges of the "hydrodynamic" variables of classical physics. These generally are integrals over suitably small volumes of densities of approximately conserved quantities such as energy, momentum, numbers of different species, etc.

The entropy of a set of histories consisting of alternatives $\left\{P_{\alpha}(t)\right\}$ at a single moment of time is

$$
\begin{aligned}
S\left(\left\{P_{\alpha}(t)\right\}\right)= & -\sum_{\alpha} p(\alpha, t) \log p(\alpha, t) \\
& +\sum_{\alpha} p(\alpha, t) \log \operatorname{Tr}\left[P_{\alpha}(t)\right]
\end{aligned}
$$

where $p(\alpha, t)=\| P_{\alpha}(t)|\Psi\rangle \|^{2}$. This coincides with the usual entropy of physical chemistry and physics when the $P_{\alpha}(t)$ are projections on the hydrodynamic quasiclassical variables described above [15]. It is low initially for the state of our universe and therefore has a tendency to increase with $t$ afterward. That is the second law of thermodynamics.

Records of history in the quasiclassical realm are often created in irreversible processes where the entropy defined above increases $^{6}$. An impact crater on the Moon, an ancient fission track in mica, a darkened photographic grain, and the printing of ink on this paper are all examples. Consistent with the

\footnotetext{
${ }^{5}$ There are time-neutral formulations of medium decoherence involving both initial and final conditions, e.g. [11].

${ }^{6}$ Increase in entropy is not necessary to create a record as in reversible computation [16].
} 
second law, records of events in history are more likely to be at the end of history furthest from an initial condition of low entropy rather than at the beginning.

The existence of records is another way of characterizing medium decoherence as discussed in Section III.A. The second law suggests that (3.6) holds in the form given and not with $C_{\alpha} R_{\beta}$ on the left-hand side. This leads to the medium decoherence of $C_{\alpha}$ but not the set $\left\{C_{\alpha}^{\dagger}\right\}$ with the projections in the inverse order. Both have the same linear positive probabilities, but only one order is connected with medium decoherence. Irreversible creation of records is therefore one reason why the quantum mechanical arrow of time is consistent with the thermodynamic arrow of time, at least for the quasiclassical realm.

\section{EXAMPLES OF LINEAR POSITIVITY}

This section calculates the candidate probabilities $p(\alpha)=$ $\operatorname{Re}\left\langle\Psi\left|C_{\alpha}\right| \Psi\right\rangle$ for a number of simple examples of physical systems, states $|\Psi\rangle$, and sets of histories $\left\{C_{\alpha}\right\}$. The intent is not to be exhaustive but to illustrate linearly positive sets of histories that are not necessarily decoherent. It's not necessary to study all of these examples to understand the general discussion of quantum theory that follows.

\section{A. The Two-slit Experiment}

We begin with the classic two-slit experiment shown in Fig. 1. Electrons from a source $S$ travel toward a vertical screen with two horizontal slits each a distance $d / 2$ from the axis perpendicular to the screen through $S$. The electrons are later detected at a second screen, parallel to the first, a distance $D$ away. A coordinate $y$ measures the vertical distance from the axis to the point of detection.

We make the usual idealizations, in particular assuming that the electrons are initially in narrow wave packets moving in the horizontal direction $x$, so that their progress in $x$ recapitulates their evolution in time. We assume that the source is far enough from the first screen that these wave packets can be analyzed into plane waves propagating in the $x$ direction with a distribution peaked about a wave number $k$. Then, to a good approximation, we can calculate the amplitudes for detection by considering just the plane wave with peak wave number $k$. That analysis follows.

At the detecting screen the amplitude that the electron travels through the upper slit $U$ to arrive at a point $y$ on the detecting screen is

$$
\Psi_{U}(y) \equiv a e^{i k S_{U}(y)} / S_{U}(y)
$$

where

$$
S_{U}(y) \equiv\left[(d / 2-y)^{2}+D^{2}\right]^{\frac{1}{2}}
$$

is the distance from the upper slit to the point on the detecting screen labeled by $y$ and $a$ is a constant amplitude. Similarly,

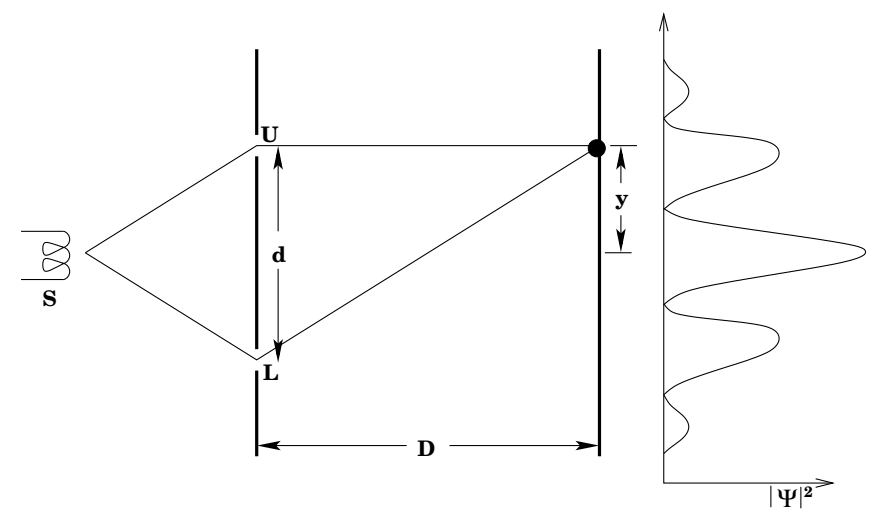

FIG. 1: The Geometry of the two-slit experiment. An electron gun at left emits an electron traveling toward a screen with two slits, its progress in space recapitulating its evolution in time. The electron is detected on the screen at right at a position $y$ with a probability density that exhibits an interference pattern. A coarse-grained set of histories for the electron can be defined by specifying which slit $(U$ or $L$ ) it went through and ranges of detected positions $y$.

the amplitude to pass through the lower slit $L$ and arrive at $y$ is

$$
\Psi_{L}(y) \equiv a e^{i k S_{L}(y)} / S_{L}(y)
$$

with

$$
S_{L}(y) \equiv\left[(d / 2+y)^{2}+D^{2}\right]^{\frac{1}{2}} .
$$

The candidate probability densities $\wp(y, U)$ and $\wp(y, L)$ to arrive at $y$ in an interval $d y$ having passed through the upper or lower slit are given by [cf. 3.1.]

$$
\begin{aligned}
& \wp(y, U)=\operatorname{Re}\left[\Psi^{*}(y) \Psi_{U}(y)\right], \\
& \wp(y, L)=\operatorname{Re}\left[\Psi^{*}(y) \Psi_{L}(y)\right] .
\end{aligned}
$$

where $\Psi(y)=\Psi_{U}(y)+\Psi_{L}(y)$. The first of these works out to be

$$
\wp(y, U)=\frac{|a|^{2}}{S_{U}}\left\{\frac{1}{S_{U}}+\frac{1}{S_{L}} \cos \left[k\left(S_{L}-S_{U}\right)\right]\right\} .
$$

The expression for $\wp(y, L)$ is the same with $L$ and $U$ interchanged. The probability density to arrive at $y$ irrespective of which slit is passed through is

$$
\begin{aligned}
\wp_{\mathrm{tot}}(y) & =\wp(y, U)+\wp(y, L), \\
& =|a|^{2}\left\{\frac{1}{S_{U}^{2}}+\frac{1}{S_{L}^{2}}+2 \frac{\cos \left[k\left(S_{L}-S_{U}\right)\right]}{S_{U} S_{L}}\right\}, \\
& =\left|\Psi_{U}(y)+\Psi_{L}(y)\right|^{2} .
\end{aligned}
$$

The last equality of course follows directly from 3.1). Figure 2 shows $\wp(y, U), \wp(y, L), \wp_{\text {tot }}(y)$ for an apposite choice of $D$ and $d$.

A set of histories of the electrons can be defined by whether the electron passed through the upper and lower slit and arrived in one or the other of an exhaustive sets of exclusive 

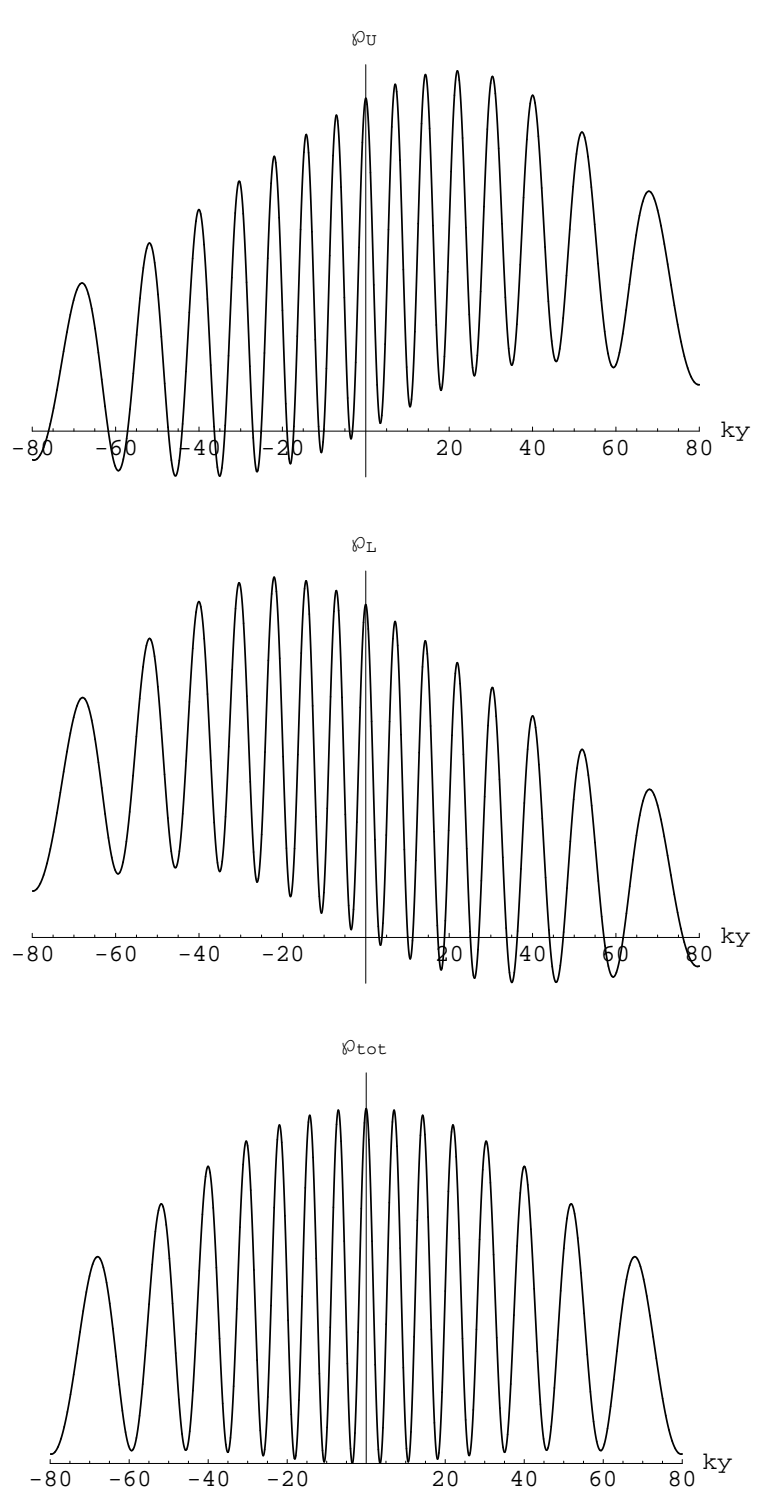

FIG. 2: Candidate probability densities for the two-slit experiment. The candidate probability density $\wp(y, U)$ to go through the upper slit and arrive at $U$ is shown at top for $k d=k D=60$ where $k$ is the wave number of the electron [cf. Fig. 1]. The corresponding candidate probability density $\wp(y, L)$ for going through the lower slit is just below. The sum $\wp_{\text {tot }}$ is the probability density to arrive at $y$ irrespective of which slit is passed through is shown at bottom. The amplitude $a$ has been assigned arbitrarily which is why the scale of the vertical axis not indicated. When these probability densities are integrated over ranges of $y$, they give candidate probabilities for the histories. The size of well-chosen ranges that yield positive probabilities is smaller than the size that would wash out the interference pattern.

ranges of $y$ of size $\left\{\Delta_{\alpha}\right\}, \alpha$ an integer. The candidate probabilities for these histories are the integrals of the densities $\wp(y, U)$ and $\wp(y, L)$ over the ranges $\left\{\Delta_{\alpha}\right\}$, e.g.

$$
p\left(\Delta_{\alpha}, U\right)=\int_{\Delta_{\alpha}} d y \wp(y, U) .
$$

Consider for simplicity the candidate probabilities illustrated in Fig. 2 with all the ranges of equal size $\Delta$. Evidently if $\Delta$ is too small, the set of histories will not be linearly positive because some of the candidate probabilities 4.8 will be negative. Equally evidently, if $\Delta$ is sufficiently large the set will be linearly positive and the $p$ 's will be probabilities. Most importantly however, the size $\Delta$ necessary to achieve positivity is smaller than that needed to wash out the interference pattern. Indeed in the limit $D \gg d$ the probability densities are

$$
\wp(y, U)=\wp(y, L) \sim \frac{|a|^{2}}{D^{2}}\left[1+\cos \left(\frac{k y d}{D}\right)\right] .
$$

Since these are both positive, arbitrarily small $\Delta$ leads to positive probabilities.

The set of histories will be medium decoherent to a good approximation where $\Delta$ is sufficiently large that the interference term averages to zero

$$
\int_{\Delta} d y \operatorname{Re}\left[\Psi_{L}^{*}(y) \Psi_{U}(y)\right] \approx 0 .
$$

That requires a $\Delta$ larger than the spacing between the interference fringes. As already mentioned, that is much larger than the $\Delta$ required for linear positivity showing concretely how linear positivity is a weaker condition than weak decoherence.

\section{B. Spin $-1 / 2$}

Consider a single free spin in a state $|\Psi\rangle$. For simplicity, assume that its Hamiltonian is zero. Consider histories specified by giving the value of the spin at one time along direction $\vec{n}_{1}$ and at a later time along a direction $\vec{n}_{2}$. Since the Hamiltonian is zero, it's not necessary to specify the particular times, only the time ordering of the alternatives matters.

Let $P_{s}^{\vec{n}}$ denote the projections on the two orientations of the spin along direction $\vec{n}$ where $s$ ranges over the possible orientations $(+,-)$. The branch state vectors are

$$
\left|\Psi_{s_{2} s_{1}}\right\rangle=P_{s_{2}}^{\vec{n}_{2}} P_{s_{1}}^{\vec{n}_{1}}|\Psi\rangle
$$

and the candidate probabilities are

$$
p\left(s_{2}, s_{1}\right)=\operatorname{Re}\left\langle\Psi \mid \Psi_{s_{2} s_{1}}\right\rangle=\operatorname{Re}\left\langle\Psi\left|P_{s_{2}}^{\vec{n}_{2}} P_{s_{1}}^{\vec{n}_{1}}\right| \Psi\right\rangle .
$$

To exhibit the candidate probabilities 4.12 explicitly it's convenient to introduce a rectangular $(x, y, z)$ coordinate system with $z$ oriented along $\vec{n}_{2}$ and $y$ chosen so that $\vec{n}_{1}$ lies in the $y-z$ plane. The angle between $\vec{n}_{1}$ and $\vec{n}_{2}$ we denote by $\delta$. In a general state $|\Psi\rangle$, the spin points along some direction which we take to be specified by polar angles $\theta$ and $\phi$ with respect to the $z$-axis. Thus in a basis in which $s_{z}$ is diagonal we can take

$$
\Psi=\left(\begin{array}{c}
e^{i \phi / 2} \cos (\theta / 2) \\
e^{-i \phi / 2} \sin (\theta / 2)
\end{array}\right) .
$$


It is then straight forward to calculate the four candidate probabilities (4.12). We find

$$
\begin{aligned}
& p(+,+)=\cos ^{2}\left(\frac{\theta}{2}\right) \cos ^{2}\left(\frac{\delta}{2}\right)+\frac{1}{4} \cos \phi \sin \theta \sin \delta \\
& p(+,-)=\cos ^{2}\left(\frac{\theta}{2}\right) \sin ^{2}\left(\frac{\delta}{2}\right)-\frac{1}{4} \cos \phi \sin \theta \sin \delta \\
& p(-,+)=\sin ^{2}\left(\frac{\theta}{2}\right) \sin ^{2}\left(\frac{\delta}{2}\right)+\frac{1}{4} \cos \phi \sin \theta \sin \delta \\
& p(-,-)=\sin ^{2}\left(\frac{\theta}{2}\right) \cos ^{2}\left(\frac{\delta}{2}\right)-\frac{1}{4} \cos \phi \sin \theta \sin \delta
\end{aligned}
$$

Note that these correctly sum to unity and that $p(+)=$ $p(+,+)+p(+,-)$, etc.

Figure 3 shows the domain of states parametrized by $(\theta, \phi)$ that imply a linearly positive set of histories for various values of the angle $\delta$. For each $\delta$ there is a significant range of states giving linearly positive histories which shrinks as $\delta$ approaches 0 or $\pi$ even though those limits correspond to linear positivity and indeed exact medium decoherence for all states. Any state with the phase $\phi=\pi / 2$ leads to a linearly positive set of histories for any $\delta$.

Almost none of these linearly positive sets of histories are medium decoherent with orthogonal branches. The conditions for medium decoherence that aren't automatically satisfied are the vanishing of the following:

$$
\begin{aligned}
\left\langle\Psi_{++} \mid \Psi_{+-}\right\rangle= & \frac{1}{4} \sin \delta[+\cos \theta \sin \delta \\
& +\sin \theta(\cos \delta \cos \phi-i \sin \phi)], \\
\left\langle\Psi_{--} \mid \Psi_{-+}\right\rangle= & \frac{1}{4} \sin \delta[-\cos \theta \sin \delta \\
& +\sin \theta(\cos \delta \cos \phi+i \sin \phi)] .
\end{aligned}
$$

Both of these vanish only for $\theta=\pi / 4$ or $3 \pi / 4$ and $\phi=0$ or $\pi$. For example as mentioned above, any state with $\phi=\pi / 2$ leads to linearly positive histories. But none of these states implies medium decoherence. Linear positivity is evidently a much weaker condition.

\section{The Three-Box Example}

The three-box example introduced by Aharonov and Vaidman [19] for other purposes has proved useful for illustrating the nature of quantum reality in consistent histories quantum mechanics [20, 21]. We use it here to illustrate the scope of the linear positivity condition.

Consider a particle that can be in one of three boxes, $A, B$, $C$ in corresponding orthogonal states $|A\rangle,|B\rangle$, and $|C\rangle$. For simplicity, take the Hamiltonian to be zero, and suppose the system to initially be in the state

$$
|\Psi\rangle \equiv \frac{1}{\sqrt{3}}(|A\rangle+|B\rangle+|C\rangle) .
$$

Consider further a state $|\Phi\rangle$ defined by

$$
|\Phi\rangle \equiv \frac{1}{\sqrt{3}}(|A\rangle+|B\rangle-|C\rangle)
$$
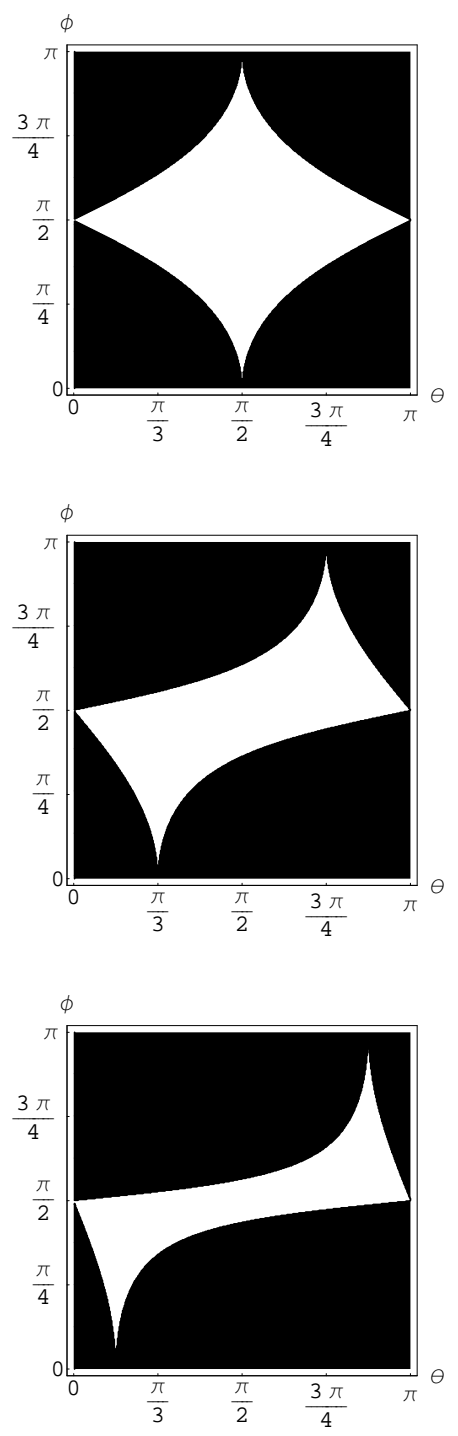

FIG. 3: Candidate probabilities for two different spin directions of a spin-1/2 particle. States of a spin-1/2 system can be labeled by two angles $(\theta, \phi)$ as in 4.13). These plots are concerned with the candidate probabilities for histories specified by values of the spin along a direction $\vec{n}_{1}$ followed (or preceded) immediately by a value of the spin along a second direction $\vec{n}_{2}$ making an angle $\delta$ with respect to the first. The plots show the states for which this set of histories is linearly positive (white) and those for which some candidate probabilities are negative (black) for given $\delta$. Only the partial range $0<\phi<\pi$ is shown because the rest can be determined from the symmetries of 4.14. From top to bottom the values of $\delta$ are $\pi / 2$, $\pi / 4$, and $\pi / 8$. For example, states with $\phi=\pi / 2$ imply these sets are linear positive for any value of $\delta$ but none is medium decoherent.

and denote the projection operators on $|\Phi\rangle,|A\rangle,|B\rangle,|C\rangle$ by $P_{\Phi}, P_{A}, P_{B}, P_{C}$ respectively. Denote with $\bar{A}$ the negation of $A$ ("not in box $A$ ") represented by the projection $P_{\bar{A}}=I-P_{A}$. The negations $\bar{\Phi}, \bar{B}, \bar{C}$ and their projections $P_{\bar{\Phi}}, P_{\bar{B}}$, and $P_{\bar{C}}$ are similarly defined. We consider sets of histories, all of which specify whether the particle is in state $|\Phi\rangle$ or not at a final 
time and in various boxes at an intermediate time after the initial one. The exact values of these times is unimportant since $H=0$. Only the order matters - initial, intermediate, and final.

An example is supplied by histories which specify whether the particle is in box $A$ or not at the intermediate time. There are four alternative histories represented by the class operators

$$
P_{\Phi} P_{A}, P_{\Phi} P_{\bar{A}}, P_{\bar{\Phi}} P_{A}, P_{\bar{\Phi}} P_{\bar{A}}
$$

Their candidate probabilities are given by 3.1 e.g.

$$
p(\Phi, A)=\operatorname{Re}\left\langle\Psi\left|P_{\Phi} P_{A}\right| \Psi\right\rangle .
$$

A little calculation shows that

$$
\begin{array}{cc}
p(\Phi, A)=1 / 9, & p(\bar{\Phi}, A)=2 / 9 \\
p(\Phi, \bar{A})=0, & p(\bar{\Phi}, \bar{A})=2 / 3
\end{array}
$$

This is a linearly positive set of histories since all the numbers (4.20) are positive or zero. Indeed, this set of histories is medium because the branch state vectors obtained by applying the class operators 4.18 to $|\Psi\rangle[c f .(3.3]$ are all orthogonal. The positivity of probabilities follows just from that.

Next consider the finer-grained set of histories which specifies whether or not the particle is in box $A$ and whether or not it is in box $B$ at the intermediate time. The eight class operators are

$$
P_{\Phi} P_{A} P_{B}, P_{\Phi} P_{A} P_{\bar{B}}, P_{\Phi} P_{\bar{A}} P_{B}, \cdots, \text { etc }
$$

This set of histories is not medium decoherent. The candidate probabilities are

$$
\begin{aligned}
p(\Phi, A, B) & =p(\bar{\Phi}, A, B)=0 \\
p(\Phi, A, \bar{B}) & =p(\Phi, \bar{A}, B)=1 / 9 \\
p(\Phi, \bar{A}, \bar{B}) & =-1 / 9 \\
p(\bar{\Phi}, A, \bar{B}) & =p(\bar{\Phi}, \bar{A}, B)=2 / 9 \\
p(\bar{\Phi}, \bar{A}, \bar{B}) & =4 / 9 .
\end{aligned}
$$

The one negative number, $p(\Phi, \bar{A}, \bar{B})=-1 / 9$, shows that this non-decoherent set of histories is also not linearly positive.

A seeming contradiction occurs [20] when one calculates the conditional probabilities for the particle to be in box $A$ or $B$ given that it is in state $\Phi$ at the later time. These are

$$
p(A \mid \Phi)=\frac{p(\Phi, A)}{p(\Phi)}, \quad p(B \mid \Phi)=\frac{p(\Phi, B)}{p(\Phi)}
$$

The symmetry between $A$ and $B$ in the definitions of $|\Psi\rangle$ and $|\Phi\rangle$ in (4.16) and 4.17) implies $p(A \mid \Phi)$ and $p(B \mid \Phi)$ are equal. Calculation shows that they are both unity. But this would seem to be a contradiction because being in $A$ and being in $B$ are exclusive alternatives: $P_{A} P_{B} \equiv 0$ implying $p(A, B)=p(A, B \mid \Phi)=0$ from 3.1. From that one would like to infer from $p(A \mid \Phi)=1$ that $p(B \mid \Phi)=0$. In fact, this inference cannot be drawn [21] and there is no contradiction. Let us see how this is established in the present context.
To calculate $p(B \mid \Phi)$ from $p(A \mid \Phi)$ and $p(A, B \mid \Phi)=0$, we need the finer-grained set of histories (4.22) referring both to box $A$ and $B$ and to $\Phi$. In this set it follows from (2.4b that

$$
\begin{aligned}
& p(A \mid \Phi)=p(A, B \mid \Phi)+p(A, \bar{B} \mid \Phi) \\
& p(\bar{A} \mid \Phi)=p(\bar{A}, B \mid \Phi)+p(\bar{A}, \bar{B} \mid \Phi)
\end{aligned}
$$

Similarly,

$$
\begin{aligned}
& p(B \mid \Phi)=p(A, B \mid \Phi)+p(\bar{A}, B \mid \Phi), \\
& p(\bar{B} \mid \Phi)=p(A, \bar{B} \mid \Phi)+p(\bar{A}, \bar{B} \mid \Phi) .
\end{aligned}
$$

If $p(A \mid \Phi)=1$ then $p(\bar{A} \mid \Phi)=0$. Were the $p$ 's positive probabilities, equating $4.24 \mathrm{~b}$ to zero would imply that both $p(\bar{A}, B \mid \Phi)$ and $p(\bar{A}, \bar{B} \mid \Phi)$ were zero. Inserting the first of these in 4.25a along with $p(A, B \mid \Phi)=0$ from 4.22a gives $p(B \mid \Phi)=0$.

But the candidate $p$ 's are not positive probabilities because the set of histories 4.21) is not linearly positive and the inference cannot be drawn. In particular from 4.22

$$
\begin{array}{ll}
p(A, B \mid \Phi)=0, & p(A, \bar{B} \mid \Phi)=1 \\
p(\bar{A}, B \mid \Phi)=1, & p(\bar{A}, \bar{B} \mid \Phi)=-1
\end{array}
$$

The sum rules 4.24 and 4.25 are exactly satisfied with $p(A \mid \Phi)=p(B \mid \Phi)=1$.

\section{A Single Particle}

The next example is more realistic. We consider a single free non-relativistic particle of mass $M$ moving in one dimension $x$. The Hamiltonian is $H=p^{2} / 2 M$. For the initial state, we choose a Gaussian wave packet of width $\sigma$ centered about the origin with zero expected value for the momentum. Specifically,

$$
\Psi(x)=\left(2 \pi \sigma^{2}\right)^{-\frac{1}{4}} e^{-x^{2} / 4 \sigma^{2}}
$$

We consider histories defined by exhaustive sets of position intervals at the initial time $t=0$ and at a later time, $t=\tau$. For simplicity we take the set of intervals $\left\{\Delta_{\alpha}\right\}$ ( $\alpha$ an integer) to be the same at both times. A coarse-grained history is defined by the pair of position intervals $\Delta_{\alpha_{1}}$ and $\Delta_{\alpha_{2}}$ the particle passes through at the two times. The candidate probabilities for these histories are denoted by $p\left(\alpha_{2}, \alpha_{1}\right)$ and constructed by implementing 3.1 as follows:

Let $P_{\alpha}(t)$ denote the Heisenberg picture projection onto the range $\Delta_{\alpha}$ of $x$ at time $t$. The candidate probabilities for the histories described above are [cf. (2.2)]

$$
p\left(\alpha_{2}, \alpha_{1}\right)=\operatorname{Re}\left\langle\Psi\left|P_{\alpha_{2}}(\tau) P_{\alpha_{1}}(0)\right| \Psi\right\rangle
$$

For purposes of computation, it is convenient to express the right-hand side of (4.28) in the Schrödinger picture. The connection is made through

$$
P_{\alpha}(t)=e^{i H t / \hbar} \hat{P}_{\alpha} e^{-i H t / \hbar}
$$


where $\hat{P}_{\alpha}$ denotes the Schrödinger picture projection onto the range $\Delta_{\alpha}$ of $x$. The result for $p\left(\alpha_{2}, \alpha_{1}\right)$ is

$$
\operatorname{Re} \int_{\Delta_{\alpha_{2}}} d x_{2} \int_{\Delta_{\alpha_{1}}} d x_{1} \Psi^{*}\left(x_{2}, \tau\right) K\left(x_{2}, x_{1}, \tau\right) \Psi\left(x_{1}, 0\right)
$$

Here $\Psi\left(x_{2}, \tau\right)$ is the initial state 4.27) evolved to time $\tau$ and $K\left(x_{2}, x_{1}, \tau\right)$ is the free particle propagator given by

$$
\begin{aligned}
K\left(x_{2}, x_{1}, \tau\right) & \equiv\left\langle x_{2}\left|e^{-i H \tau / \hbar}\right| x_{1}\right\rangle \\
& =\left(\frac{M}{2 \pi i \hbar \tau}\right)^{\frac{1}{2}} \exp \left[-\frac{M\left(x_{2}-x_{1}\right)^{2}}{2 \hbar \tau}\right] .
\end{aligned}
$$

The evolved Gaussian wave packet 4.27 is

$$
\begin{aligned}
\Psi(x, \tau)= & \frac{1}{\left(2 \pi \sigma^{2}\right)^{\frac{1}{4}}}\left(1+\frac{\hbar \tau}{2 \sigma^{2} M}\right) \\
& \times \exp \left[-\frac{x^{2}}{4 \sigma^{2}}\left(1+\frac{\hbar \tau}{2 \sigma^{2} M}\right)^{-1}\right] .
\end{aligned}
$$

The expression for the candidate probabilities 4.30 simplifies considerably if we restrict attention to times which are short compared to the wave packet spreading time

$$
\tau \ll \tau_{\text {spread }} \equiv \frac{2 \sigma^{2} M}{\hbar} \approx\left(2 \times 10^{27} \mathrm{~s}\right)\left(\frac{\sigma}{1 \mathrm{~cm}}\right)^{2}\left(\frac{M}{1 \mathrm{gm}}\right) .
$$

There are many interesting situations in which this is a realistic assumption. In this approximation

$$
p\left(\alpha_{2}, \alpha_{1}\right)=\left(\frac{1}{2 \pi \sigma^{2}}\right)^{\frac{1}{2}}\left(\frac{M}{2 \pi \hbar \tau}\right)^{\frac{1}{2}} \int_{\Delta_{\alpha_{2}}} d x_{2} \int_{\Delta_{\alpha_{1}}} d x_{1} \exp \left[-\frac{\left(x_{2}^{2}+x_{1}^{2}\right)}{4 \sigma^{2}}\right] \cos \left[\frac{M\left(x_{2}-x_{1}\right)^{2}}{2 \hbar \tau}-\frac{\pi}{4}\right] .
$$

We now discuss the behavior of these candidate probabilities for a simple coarse graining of this set of histories.

The probabilities for the coarse graining we consider answer the question: Does the particle remain at the origin or does it move elsewhere? Specifically, we consider the set consisting of just two histories: The history $L$ in which the particle is localized in an interval $\Delta$ centered on the origin at both times $t_{1}$ and $t_{2}$, and the history $\bar{L}$ in which it is not.

The candidate probability $p_{L}(\Delta)$ for the particle to be localized at both times is given by 4.34 with $\Delta_{\alpha_{1}}=\Delta_{\alpha_{2}} \equiv \Delta$. The candidate probability $p_{\bar{L}}(\Delta)$ that is not so localized, can be found from this and

$$
p_{L}(\Delta)+p_{\bar{L}}(\Delta)=1
$$

Equation (4.34) can be organized to give a tractable expression for $p_{L}(\Delta)$ by defining new integration variables

$$
X=\left(x_{1}+x_{2}\right) / 2, \quad \xi=x_{2}-x_{1},
$$

and introducing a characteristic wavelength of oscillation

$$
\lambda \equiv\left[4 \pi\left(\frac{\hbar \tau}{M}\right)\right]^{\frac{1}{2}}=1.1 \times 10^{-13}\left(\frac{\tau}{1 s}\right)^{\frac{1}{2}}\left(\frac{1 \mathrm{~g}}{M}\right)^{\frac{1}{2}} \mathrm{~cm}
$$

Then, after some algebra

$$
p_{L}(\Delta)=\sqrt{\frac{2}{\pi \sigma^{2}}} \int_{0}^{\Delta} d X e^{-X^{2} / 2 \sigma^{2}} J(\Delta-X)
$$

where

$$
\begin{aligned}
J(z) & \equiv \frac{2^{\frac{3}{2}}}{\lambda} \int_{0}^{z} d \xi e^{-\xi^{2} / 8 \sigma^{2}} \cos \left[2 \pi\left(\frac{\xi}{\lambda}\right)^{2}-\frac{\pi}{4}\right] \\
& =\operatorname{Re}\left\{\operatorname{Erf}\left[\sqrt{\pi}(1-i) \frac{z}{\lambda}\right]\right\} .
\end{aligned}
$$

These expressions are quoted in the approximation $\lambda \ll \sigma$ typically valid for large particles as 4.37) shows. The general ones are not much more complicated.

As $\Delta$ becomes large, $p_{L}(\Delta)$ must approach unity and 4.38 exhibits this explicitly $(\operatorname{Erf}(\infty)=1)$. But also from 4.28, and the general result $3.2, p_{L}(\Delta) \leq 1$. For sufficiently large $\Delta$ this set of histories is linearly positive. Analysis of the situation for $\Delta \lesssim \sigma$ requires numerical integration of (4.38) but the following result emerges: At least for $\lambda / \sigma \sim 1 / 100, p_{L}(\Delta)$ is positive over the whole range of $\Delta$ from 0 to $\infty$. Figure 4 shows $p_{L}(\Delta)$ for this case. Taken together with $p_{L}(\Delta) \leq 1$, this means that this set is always positive. The quantities $p_{L}(\Delta)$ and $p_{\bar{L}}(\Delta)$ are genuine probabilities.

Although linear positive, this set of histories is far from decoherent. With $C_{L} \equiv P_{\Delta}(\tau) P_{\Delta}(0)$ the off-diagonal elements of the decoherence functional are

$$
\begin{aligned}
D(L, \bar{L}) & \equiv\left\langle\Psi\left|C_{L}^{\dagger} C_{\bar{L}}\right| \Psi\right\rangle=\left\langle\Psi\left|C_{L}^{\dagger}\left(I-C_{L}\right)\right| \Psi\right\rangle \\
& =\left\langle\Psi\left|P_{\Delta}(0) P_{\Delta}(\tau) P_{\bar{\Delta}}(0)\right| \Psi\right\rangle
\end{aligned}
$$

where $P_{\bar{\Delta}}$ is the projection on the range outside $\Delta$ at $t=0$. We can think of 4.39) as the overlap of two states $\left|\Psi_{L}\right\rangle \equiv$ $P_{\Delta}(\tau) P_{\Delta}(0)|\Psi\rangle$ and $\left|\Psi_{\bar{L}}\right\rangle \equiv P_{\Delta}(\tau) P_{\bar{\Delta}}(0)|\Psi\rangle$. When $\Delta \gg \sigma$, 


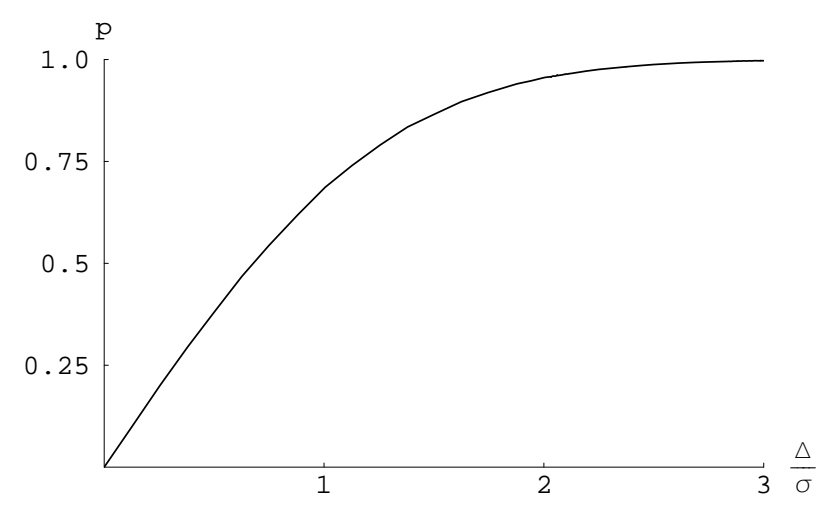

FIG. 4: Candidate probabilities for a single free particle in a stationary Gaussian wave packet to remain in a position interval $\Delta$ after a time short compared to the wave-packet spreading time. The plot shows the candidate probability $p_{L}(\Delta)$ defined by 4.38a for $\lambda / \sigma=10^{-2}$. This is positive over the whole of the range $\Delta$ indicating that the set consisting of the history in which the particle remains localized and the history where does not so remain is linearly positive.

$\left|\Psi_{\bar{L}}\right\rangle$ will have negligible length because $P_{\bar{\Delta}}(0)|\Psi\rangle$ is negligible. Consider therefore $\Delta \ll \sigma$. The wave function of $P_{\bar{\Delta}}(0)|\Psi\rangle$ consists of those parts of the initial packet 4.27) outside of $\Delta$. Because of the sharp interior edges, this evolves rapidly to fill in the center around $x=0$. After projection of $P_{\Delta}(\tau)$ there is significant overlap with the wave function of $\left|\Psi_{L}\right\rangle$ of order $(\Delta / \sigma)$ and thus absence of decoherence.

In general we expect the action of an environment to be necessary to carry away the phases between such alternatives and make them decoherent (e.g. [14]). Despite the absence of an environment and its consequent decoherence this set of histories is linearly positive in the approximations considered.

\section{E. A Spacetime Alternative}

The familiar example of a coarse-grained history is a sequence of events at a series of definite times. But decoherent histories quantum theory permits more general coarse grainings that extend continuously over ranges of time [22, 23]. Such spacetime coarse grainings may provide more realistic models of measurement processes that extend over time. Analogous coarse grainings may be essential for a quantum theory of gravity where there is no fixed notion of time (e.g. [9]).

To illustrate the idea of a spacetime coarse graining, consider the motion of a single free particle of mass $M$ moving in one dimension $x$ with Hamiltonian $H=p^{2} / 2 M$ over a range of times from 0 to $T$. The set of fine-grained histories of the particle's motion consists of the paths $x(t)$ on the interval $[0, T]$. Sets of alternative coarse-grained histories are defined by partitions of these fine-grained histories into mutually exclusive classes $c_{\alpha}, \alpha=1,2, \cdots$. Each class is a coarse-grained history.

The class operators $C_{\alpha}$ for these coarse-grained histories can be constructed from sums over their constituent fine- grained histories. To see how, first consider a partition of the paths by an exhaustive set of position intervals $\left\{\Delta_{\alpha}\right\}$ at a series of times $t_{1}<\cdots<t_{n}$. The class operator in the Heisenberg picture is $C_{\alpha}=P_{\alpha_{n}}\left(t_{n}\right) \cdots P_{\alpha_{1}}\left(t_{1}\right)$ where $P_{\alpha}(t)$ is the projection on the range $\Delta_{\alpha}$ at time $t$. Matrix elements of the $C_{\alpha}$ in the Heisenberg picture (HP) can be transformed into transition amplitudes in Schrödinger picture (SP), and these transition amplitudes can be expressed as path integrals. The result is

$$
\begin{aligned}
\left\langle\Phi\left|C_{\alpha}\right| \Psi\right\rangle_{\mathrm{HP}} & =\left\langle\Phi(T)\left|\hat{C}_{\alpha}\right| \Psi(0)\right\rangle_{\mathrm{SP}}, \\
& =\int_{c_{\alpha}} \delta x \Phi^{*}\left(x_{T}, T\right) e^{i S[x(t)] / \hbar} \Psi\left(x_{0}, 0\right) .
\end{aligned}
$$

Here,

$$
\hat{C}_{\alpha} \equiv e^{-i H T / \hbar} C_{\alpha}
$$

and the path integral is over all paths $x(t)$ in the class $c_{\alpha}$ including an integral over the end points $x_{0}$ and $x_{T}$. (More details on establishing this relation can be found in [22] and [24].) The candidate probabilities for the coarse-grained histories are, from 3.1

$$
p(\alpha)=\operatorname{Re}\left\langle\Psi(T)\left|\hat{C}_{\alpha}\right| \Psi(0)\right\rangle_{\mathrm{SP}}
$$

From now on we drop the SP's and HP's and rely on context to distinguish the two pictures.

The derivation sketched above of the connection 4.40 between matrix elements of class operators and path integrals over classes was for coarse grainings by ranges of position at a sequence of times. But the result motivates using path integrals to define class operators for arbitrary partitions of the fine-grained histories $x(t)$ into mutually exclusive classes including partitions defining alternatives extending over a range of time. Eq. 4.42 gives candidate probabilities for these spacetime coarse grainings.

A simple model illustrates the idea [22, 25]. Partition all paths $x(t)$ on the interval $[0, T]$ into the two classes

$$
\begin{aligned}
& R: \text { paths } x(t) \text { that always remain in the region } \\
& x>0 \text { between times } 0 \text { and } T \text {. } \\
& \bar{R} \text { : paths } x(t) \text { that sometimes are in the region } \\
& x<0 \text { between times } 0 \text { and } T \text {. }
\end{aligned}
$$

Evidently, these classes are exhaustive and mutually exclusive. $R$ here means "right", $x>0$, and $p_{R}$ computed from (4.42) is the probability that the particle remains at $x>0$ for the whole time interval and never crosses into $x<0$.

A sum over all paths of the form (4.40) that is restricted to $x>0$ is the same as an unrestricted sum in the presence of an infinite potential barrier at values of $x<0$ [22]. Let $H_{R}$ denote the Hamiltonian of the particle including this barrier. The branch state vector for the class $R$ can be written

$$
\left|\Psi_{R}(T)\right\rangle=P_{R} e^{-i H_{R} T / \hbar} P_{R}|\Psi(0)\rangle
$$

where $P_{R}$ is the projection onto $x>0$. The candidate probability $p_{R}$ is [cf. 4.42]

$$
p_{R}=\operatorname{Re}\left\langle\Psi(T) \mid \Psi_{R}(T)\right\rangle \text {. }
$$


It's not necessary to derive a separate expression for $p_{\bar{R}}$ in this simple example. From (1.1), (4.39) and (4.40) it follows that

$$
p_{R}+p_{\bar{R}}=1
$$

(whether or not the set is linearly positive.) Calculation of $p_{R}$ will therefore determine $p_{\bar{R}}$.

For explicit calculation it is convenient to rewrite (4.44) in terms of wave functions. Let $\Psi(x, 0)$ denote the wave function of the initial state and $\Psi_{U}(x, T)$ its unrestricted evolution under $H$. Let $\Phi(x, 0) \equiv P_{R} \Psi(x, 0)$, and denote by $\Phi_{R}(x, T)$ and $\Phi_{U}(x, T)$ its evolution under $H_{R}$ and $H$ respectively. Evolution in the presence of an infinite barrier is especially simple, and

$$
\Phi_{R}(x, T)=\Phi_{U}(x, T)-\Phi_{U}(-x, T), x>0 .
$$

Thus, we can write

$$
\begin{aligned}
p_{R} & =\operatorname{Re} \int_{0}^{\infty} d x \Psi_{U}^{*}(x, T) \Phi_{R}(x, T) \\
& =\operatorname{Re} \int_{0}^{\infty} d x \Psi_{U}^{*}(x, T)\left[\Phi_{U}(x, T)-\Phi_{U}(-x, T)\right] .
\end{aligned}
$$

This form allows both quantitative computation and qualitative discussion. Since

$$
\|\left|\Phi_{R}(T)\right\rangle\|=\|\left|\Phi_{R}(0)\right\rangle\|=\|\left|P_{R} \Psi(0)\right\rangle \|,
$$

an immediate consequence of 4.47) is

$$
p_{R} \leq \| P_{R}\left|\Psi_{U}(T)\right\rangle\|\| P_{R}|\Psi(0)\rangle \| .
$$

This shows $p_{R} \leq 1$ and $p_{\bar{R}} \geq 0$.

We now evaluate 4.47) for a very simple initial wave function $\Psi(x, 0)$. We consider a Gaussian wave packet of width $\sigma$ centered about an initial position $X_{0}>0$ and moving toward negative $x$ with a (negative) momentum $K_{0}$. Specifically, assume

$$
\Psi(x, 0)=\left(2 \pi \sigma^{2}\right)^{-\frac{1}{4}} e^{i K_{0} x} e^{-\left(x-X_{0}\right)^{2} /\left(4 \sigma^{2}\right)} .
$$

To keep expressions simple, we use units when $\hbar=1$ as we will for the remainder of this section.

The following approximations make the evaluation of the integral 4.47) for $p_{R}$ straightforward.

1. We assume that

$$
X_{0} \gg \sigma
$$

so that the initial wave function is negligible for $x<0$, i.e.

$$
\Phi(x, 0) \equiv P_{R} \Psi(x, 0) \approx \Psi(x, 0) .
$$

2. We assume that the time $T$ is short compared to the time over which the wave packet spreads significantly, specifically

$$
T \ll 2 \sigma^{2} M .
$$

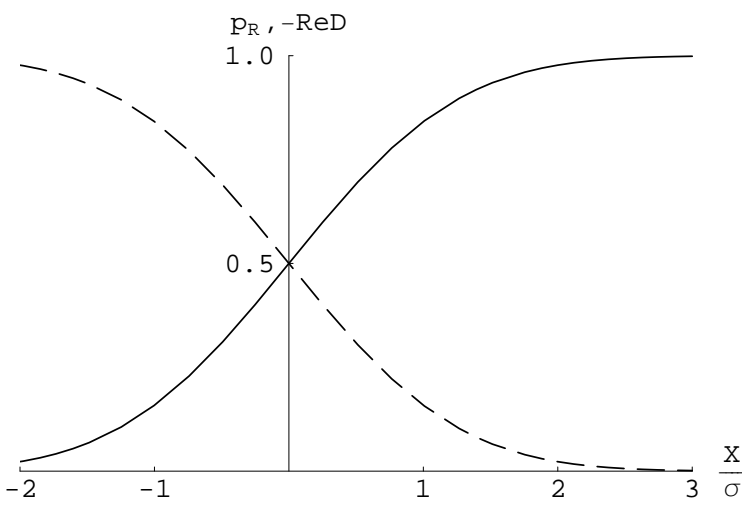

FIG. 5: Candidate probabilities for a spacetime coarse graining. A free particle moving in one dimension is initially in a Gaussian wave packet of width $\sigma$ traveling toward negative $x$ with a momentum $K_{0}=-20 / \sigma$ in $\hbar=1$ units. The solid curve shows the candidate probability $p_{R}$ for the particle to remain always at positive $x$ over a time $T$ short compared to the wave packet spreading time. This is plotted as a function of the final position of the packet's center $X$ that is connected to the initial position $X_{0}$ and $T$ by 4.54). The probability is positive over the entire range computed showing that the set consisting of the history $(R)$, where the particle remains at positive $x$, and the history $(\bar{R})$, where it does not, is linearly positive. The dashed curve shows the real part of the overlap $\left\langle\Psi_{R} \mid \Psi_{\bar{R}}\right\rangle$ which must vanish for this set of histories to be medium decoherent. Evidently there is a significant range of linearly positive sets which are not medium decoherent.

With these two approximations, the shape of the wave packet remains approximately unchanged under evolution over the time $T$. Only the center shifts to the value

$$
X=X_{0}+K_{0} T / M \text {. }
$$

Specifically,

$$
\begin{aligned}
\Phi_{U}(x, T) & \approx \Psi_{U}(x, T) \\
& \approx\left(2 \pi \sigma^{2}\right)^{-\frac{1}{4}} e^{i K_{0} x} e^{-(x-X)^{2} / 4 \sigma^{2}} e^{-i\left(K_{0}^{2} / 2 M\right) T} .
\end{aligned}
$$

The integral 4.47) for $p_{R}$ is then

$$
\begin{aligned}
p_{R} & \approx\left(2 \pi \sigma^{2}\right)^{-\frac{1}{2}} \int_{0}^{\infty} d x e^{-(x-X)^{2} / 2 \sigma^{2}} \\
& \times\left[1-e^{-x X / \sigma^{2}} \cos \left(2 K_{0} x\right)\right]
\end{aligned}
$$

which is straightforward to evaluate numerically.

Assumption (4.51) means that $K_{0}$ must be large for the center of the wave packet to reach the neighborhood of $x=0$ in the times limited by (4.53). Specifically, $K_{0} \sigma \gg 1$. Otherwise, the wave packet remains to the right of $x=0$ and $p_{R} \approx 1$.

Figure 5 shows $p_{R}$ from (4.56) as a function of the position of the center $X$ at time $T$ for $K_{0}=-20 / \sigma$. Only values of $X \gtrsim-2$ are shown for which $X_{0} \gtrsim 3 \sigma$ in a time $T$ about $1 / 10$ of the spreading time in 4.53). For smaller values of $X$, the approximation 4.55) would be inaccurate.

For large values of $X$, the wave packet is localized some place in $X>0$ over the whole of the time interval. We might 
therefore expect $p_{R} \approx 1$, and it is. For smaller $X$, there is a significant probability of crossing $X=0$ at least once in the time interval 0 to $T$. The important point is that, over the whole of the calculated range, $p_{R}$ lies between 0 and 1. Likewise for $p_{\bar{R}}$ from 4.43. The set of alternatives is thus linearly positive with genuine probabilities. We now turn to the question of whether this set of alternatives decoheres.

The set of coarse-grained histories $R$ and $\bar{R}$ decoheres when [cf. (1.4)]

$$
D(R, \bar{R}) \equiv\left\langle\Psi_{R}(T) \mid \Psi_{\bar{R}}(T)\right\rangle \approx 0
$$

The branch state vector $\left|\Psi_{R}\right\rangle$ was exhibited in (4.43). Relation (1.1) and (4.41) imply that

$$
\left|\Psi_{\bar{R}}(T)\right\rangle=|\Psi(T)\rangle-\left|\Psi_{R}(T)\right\rangle .
$$

In terms of wave functions

$$
D(R, \bar{R})=\int_{0}^{\infty} d x \Phi_{R}^{*}(x, T)\left[\Psi_{U}(x, T)-\Phi_{R}(x, T)\right]
$$

where $\Phi_{R}(x, T)$ can be taken to be given by (4.46. In the approximation where 4.52 holds, this is

$$
D(R, \bar{R}) \approx \int_{0}^{\infty} d x\left[\Psi_{U}^{*}(x, T)-\Psi_{U}^{*}(-x, T)\right] \Psi_{U}(-x, T) .
$$

Figure 5 shows $\operatorname{Re} D(R, \bar{R})$ calculated from 4.60) plotted against $X$. For large positive $X$, the particle remains on the right. There is thus essentially only one history with significant probability, $\left|\Psi_{R}\right\rangle \approx|\Psi\rangle,\left|\Psi_{\bar{R}}\right\rangle \approx 0$ and decoherence is automatic. For large negative $X$, almost all of the wave packet $\Psi_{U}(x, T)$ will have crossed $x=0$. That is outside the range where (4.60) is necessarily a good approximation, but the first term will vanish and $\left\langle\Psi_{R} \mid \Psi_{\bar{R}}\right\rangle \approx-1$. That is far from decoherence.

Figure 5 shows that over a wide range of situations for which this set of histories is linearly positive, it is not decoherent. That is consistent with linear positivity being a weaker condition for probabilities.

\section{VIRTUAL PROBABILITIES}

What about sets of histories that are not linearly positive? Can the candidate probabilities $p(\alpha)$ defined by 3.1 be put to use even if they are outside the range 0 to 1 ? This section will show that they can. In particular, $p(\alpha)$ outside the range 0 to 1 can be employed as intermediate steps in the calculation of probabilities that do lie between 0 and 1 . That is because probability sum rules like 2.4b are satisfied by the $p(\alpha)$ defined by 2.1 whether or not they lie between 0 and 1 as a consequence of 2.3 . For this reason, if some $p(\alpha)$ in a set are outside the range 0 to 1 , we say the set has virtual probabilities. When a contrasting term is needed for the case when all the $p(\alpha)$ are between 0 or 1 , we say the set has real probabilities. $^{7}$ "Real" in this context is thus a synonym for linearly positive. Extending sets of probabilities to include virtual values provides a simple and unified approach to the quantum mechanics of closed systems as we shall describe in Section VI. Anticipating this extension, in this Section we refer to the candidate probabilities $p(\alpha)$ defined by (3.1) as "probabilities" whether or not they are real or virtual.

To understand what virtual probabilities might mean, first consider what they cannot mean. Evidently, virtual probabilities cannot coincide with the relative frequencies of repeated events such as the probabilities of the outcomes of identical measurements on an ensemble of identical subsystems. More generally, alternatives with virtual probabilities cannot be exactly recorded or measured. The exact correlation between alternative values of records represented by projections $\left\{R_{\beta}\right\}$ and histories represented by class operators $\left\{C_{\alpha}\right\}$ would mean [cf. 3.6]

$$
p(\beta, \alpha) \equiv \operatorname{Re}\left\langle\Psi\left|R_{\beta} C_{\alpha}\right| \Psi\right\rangle=\delta_{\alpha \beta} p(\alpha) .
$$

Sum both sides of this relation over $\beta$ using $\sum_{\beta} R_{\beta}=I$. Sum both sides over $\alpha$ using $\sum_{\alpha} C_{\alpha}=I$. The resulting relations imply the identity

$$
p(\alpha) \equiv \operatorname{Re}\left\langle\Psi\left|C_{\alpha}\right| \Psi\right\rangle=\operatorname{Re}\left\langle\Psi\left|R_{\alpha}\right| \Psi\right\rangle
$$

showing that the probabilities of the records are the same as the probabilities of the histories. Since the $\left\{R_{\alpha}\right\}$ are projections, their probabilities must be between 0 and 1 . Sets of histories with virtual probabilities therefore cannot be exactly recorded. Since recording outcomes is usually taken to be an essential part of a measurement process $^{8}$, we can say that sets of histories with virtual probabilities cannot describe the outcomes of measurements. Extending the notion of probability to include virtual values thus does not risk assigning a virtual value to the probability of anything measured or recorded exactly. Of course, the records of realistic measurements are typically correlated with the measured history, not exactly, but rather to an excellent approximation.

Virtual probabilities can in principle be employed in the calculation of real ones as the following example illustrates. Consider the probabilities $p(\gamma \mid p d)$ of some future alternatives $\{\gamma\}$ given present data $p d$. It may be more efficient to calculate these probabilities by first determining the probabilities $p(\beta \mid p d)$ of alternatives $\{\beta\}$ in the past and then with these, and with present data, calculate the probabilities of the future alternatives $\{\gamma\}$. More concretely, it may be efficient to calculate $p(\gamma \mid p d)$ from the relation

$$
p(\gamma \mid p d)=\sum_{\beta} p(\gamma \mid p d, \beta) p(\beta \mid p d) .
$$

For example, if a current manuscript $(p d)$ records that Caesar invaded Britain in $55 \mathrm{BC}$, we might predict that other

\footnotetext{
7 To maintain the usual contrast between virtual and real, we accept the risk that "real" can be taken to be a contrast with "imaginary".

${ }^{8}$ See e.g. [26]
} 
manuscripts yet to be discovered $(\{\gamma\})$ would record the same date. However, the most direct route to that prediction would be to first infer that Caesar did invade Britain in 55 BC (one of the alternatives $\beta$ ) and from that calculate the probabilities of what other manuscripts might say. We reconstruct the past to help explain the future.

Our purpose here is not to discuss the utility of reconstructing the past ${ }^{9}$. Rather, it is to point out that (5.3) is a consequence of 2.1) and 3.1 which hold whether or not the probabilities $p(\beta \mid p d)$ are between 0 and 1. In principle, virtual $p(\beta \mid p d)$ could be used in the intermediate step in (5.3) provided the result $p(\gamma \mid p d)$ were real probabilities.

The three-box example discussed in Section IV.C provides a simple, if artificial, example. Suppose we are interested in predicting probabilities for whether the particle will be either in box $A$, or else not in $A$ (i.e., in box $B$ or $C$ ) at some future time given $\Phi$. We denote these alternatives by $A^{f}$ and $\bar{A}^{f}$. The probability $p\left(\bar{A}^{f} \mid \Phi\right)$ for instance is given by

$$
p\left(\bar{A}^{f} \mid \Phi\right)=\frac{p\left(\bar{A}^{f}, \Phi\right)}{p(\Phi)}=\frac{\operatorname{Re}\left\langle\Psi\left|\left(P_{B}+P_{C}\right) P_{\Phi}\right| \Psi\right\rangle}{\operatorname{Re}\left\langle\Psi\left|P_{\Phi}\right| \Psi\right\rangle}
$$

From (4.16) and 4.17) we find,

$$
p\left(A^{f} \mid \Phi\right)=1, \quad p\left(\bar{A}^{f} \mid \Phi\right)=0
$$

- a set of real probabilities.

But we could calculate these probabilities by first calculating the $p$ 's for the alternatives $A^{p}, B^{p}, C^{p}$ that the particle was in box $A, B$, or $C$ in the past given $\Phi$ and then using 5.3 to calculate the future probabilities for $A^{f}$ and $\bar{A}^{f}$. The probabilities for the past alternatives $A^{p}, B^{p}, C^{p}$ given $\Phi$ can be negative, for example

$$
p\left(C^{p} \mid \Phi\right)=\frac{p\left(\Phi, C^{p}\right)}{p(\Phi)}=\frac{\operatorname{Re}\left\langle\Psi\left|P_{\Phi} P_{C}\right| \Psi\right\rangle}{\operatorname{Re}\left\langle\Psi\left|P_{\Phi}\right| \Psi\right\rangle}=-1 .
$$

Similarly, $p\left(A^{p} \mid \Phi\right)=p\left(B^{p} \mid \Phi\right)=1$. Despite this the reader may easily verify that employing (5.3) with $\{\gamma\}=$ $\left\{A^{f}, \bar{A}^{f}\right\},\{\beta\}=\left\{A^{p}, B^{p}, C^{p}\right\}$ and $p d=\Phi$ gives the correct answer displayed in 5.5.

Feynman [28] explored the uses of negative probabilities in intermediate steps in a variety of circumstances in physics. These included the probabilities for position and momentum defined by the Wigner distribution, for the emission of virtual non-transverse photons in electrodynamics, in two-state systems, and in the two-slit experiment. He concluded that extending the notion of probability to negative values was useful provided these negative values are interpreted to mean that the situation is "unattainable or unverifiable". We take the same viewpoint here.

The quantum mechanics of a closed system then can be formulated as follows: Assign probabilities to all sets of alternative coarse-grained histories by $p(\alpha)=\operatorname{Re}\left\langle\Psi\left|C_{\alpha}\right| \Psi\right\rangle$. The probabilities $p(\alpha)$ may be real or virtual. Virtual probabilities

\footnotetext{
${ }^{9}$ For that see [27]
}

can be employed in the calculation of real ones. Probabilities predicted for exactly recorded histories are always real. This is a simple and general formulation whose utility we describe in the next section. Linear positivity and medium decoherence are special cases.

\section{COMPARING DECOHERENCE CONDITIONS}

Four decoherence conditions restricting the sets of alternative coarse-grained histories to which quantum mechanics predicts probabilities have been considered in this paper. In order of increased restriction (roughly inverse to the order presented) they are:

- Extended Probabilities (EP)

Assign probabilities to all sets of alternative coarsegrained histories according to

$$
p(\alpha)=\operatorname{Re}\left\langle\Psi\left|C_{\alpha}\right| \Psi\right\rangle
$$

whether they are real (between 0 and 1 ) or virtual. This is no restriction at all. Calculate the probabilities of exactly recorded alternatives, or the frequencies of repeated independent alternatives, secure in the knowledge that these will be between 0 and 1 .

- Linear Positivity (LP)

Assign probabilities only to sets of alternative coarsegrained histories for which all candidate probabilities in the set given by 6.1 lie in the range 0 to 1 of real probabilities.

- Real Linear Positivity (RLP)

Assign probabilities only to sets of alternative coarse grained histories which are linearly positive and for which

$$
\operatorname{Im}\left[\left\langle\Psi\left|C_{\alpha}\right| \Psi\right\rangle\right] \approx 0, \quad \text { all } \alpha,
$$

either exactly or approximately well beyond the standard with which the resulting probabilities are used.

- Medium Decoherence (MD)

Assign probabilities only to sets of alternative coarsegrained histories for which the branch state vectors for all histories in the set are mutually orthogonal

$$
\left\langle\Psi_{\alpha} \mid \Psi_{\alpha^{\prime}}\right\rangle \approx 0, \alpha \neq \alpha^{\prime}
$$

either exactly, or to an approximation well beyond the standard with which the resulting probabilities are used. Calculate probabilities either by 6.1 or by

$$
p(\alpha)=\|\left|\Psi_{\alpha}\right\rangle\left\|^{2}=\right\| C_{\alpha}|\Psi\rangle \|^{2}
$$

which are equivalent if medium decoherence is exact. Probabilities defined by 6.4 necessarily lie between 0 and 1. 
This concluding section compares these different decoherence conditions.

As information gathering and utilizing systems (IGUS's), we employ almost exclusively coarse grainings of the usual quasiclassical realm. By the usual quasiclassical realm, we mean roughly histories of coarse-grained alternatives defined by ranges of values of averages of densities of approximately conserved quantities (such as energy, momentum, etc.) over suitable volumes. With the initial condition and Hamiltonian of our universe, the volumes can be chosen large enough that the histories are medium decoherent and yet small enough to supply a reasonably fine-grained description of the universe over a wide range of time and distance scale. Individual histories of this realm exhibit patterns of correlations in time governed by effective classical equations of motion interrupted by frequent small quantum fluctuations and occasional major ones.

For practical purposes it might be possible to restrict the predictions of quantum theory to the usual quasiclassical realm. Indeed, in some loose sense, this was the view of founders of the subject such as Bohr and Heisenberg. But it so far has been difficult to give the usual quasiclassical realm a precise definition despite the considerable steps that have been taken in that direction [29]. For reasons of generality, convenience, and completeness it has proved useful to formulate quantum theory with weaker, less anthropocentric, but more precise conditions that allow many more sets of histories that are nothing like the usual quasiclassical realm. The three conditions discussed in this paper are examples.

Moving between EP, LP, RLP, and MD in the of increasing restriction is moving toward the usual quasiclassical realm. For example, the virtual probabilities of EP for non-equal values of a quantity commuting with the Hamiltonian at two different times may not be zero if other alternatives intervene as in 3.8. But they are zero under the more restrictive LP conditions as the discussion in Section III.C shows.

The conditions RLP and MD are consistent with the usual notions of statistical independence of identical subsystems; EP and LP are not.

The conditions EP, LP and RLP are time-neutral, but the more restrictive MD incorporates an arrow of time as discussed in Section III.C. This arrow allows individual histories to be described as a narrative in which one event is followed by another, then another, etc. Quantum theory then can be formulated in terms of an evolving state, and prediction distinguished from retrodiction. For example, consider a medium decoherent set of histories defined by alternatives $\left\{P_{\alpha_{1}}^{1}\left(t_{1}\right)\right\}$, $\left\{P_{\alpha_{2}}^{2}\left(t_{2}\right)\right\}, \cdots\left\{P_{\alpha_{n}}^{n}\left(t_{n}\right)\right\}$ at a series of times $t_{1}<t_{2}<\cdots<t_{n}$. At any intermediate time $t_{k}$, we can calculate the conditional probability $p\left(\alpha_{n}, \cdots, \alpha_{k+1} \mid \alpha_{k}, \cdots, \alpha_{1}\right)$ for future alternatives $\alpha_{k+1}, \cdots, \alpha_{n}$ given that $\alpha_{1}, \cdots, \alpha_{k}$ have already happened by

$$
\begin{aligned}
& p\left(\alpha_{n}, \cdots, \alpha_{k+1} \mid \alpha_{k}, \cdots, \alpha_{k}\right) \\
& =\| P_{\alpha_{n}}^{n}\left(t_{n}\right) \cdots P_{\alpha_{k+1}}^{k+1}\left(t_{k+1}\right)\left|\Psi_{\alpha_{k} \cdots \alpha_{1}}\right\rangle \|^{2}
\end{aligned}
$$

where

$$
\left|\Psi_{\alpha_{k} \cdots \alpha_{1}}\right\rangle=\frac{P_{\alpha_{k}}^{k}\left(t_{k}\right) \cdots P_{\alpha_{1}}^{1}\left(t_{1}\right)|\Psi\rangle}{\| P_{\alpha_{k}}^{k}\left(t_{k}\right) \cdots P_{\alpha_{1}}^{1}\left(t_{1}\right)|\Psi\rangle \|}
$$

Events to the future of $t_{k}$ can be predicted just from the state $\left|\Psi_{\alpha_{k} \cdots \alpha_{1}}\right\rangle$ representing the present. In the Heisenberg picture used here, that state is constant in time except when interrupted by the action of projections ("reductions") representing the alternatives.

The past cannot be retrodicted just from a state in the present, but requires in addition the state $|\Psi\rangle$. A similar statement holds for the future in the context of LP or EP in general (see, e.g. [12, 27]). It could not be otherwise since these formulations are time-neutral.

Histories of the usual quasiclassical realm are medium decoherent because of physical mechanisms which dissipate phases between branches [34, 35, 36] that result from the interaction between the variables followed in the usual quasiclassical coarse graining and ones ignored constituting an environment or bath in simple models. These interactions create records of the histories [37, 38] and the existence of records is a general characterization of medium decoherence as we saw in Section III.A. The restriction to medium decoherence is thus consistent with the usual quasiclassical realm.

Imposing restrictive decoherence conditions like medium and strong decoherence [30] are useful first steps in defining classicality. But the weaker conditions (LP) and (EP) also have their uses.

An example is approximate medium decoherence. As mentioned above, the branch state vectors of the usual quasiclassical realm are not expected to be exactly orthogonal, but only to an approximation good well beyond the standard to which the resulting approximate probabilities can be used. Some are uneasy about basing a fundamental formulation of quantum mechanics on approximate medium decoherence [39]. Is there a formulation of the quantum mechanics of closed systems free from any approximate notion of decoherence for realistic coarse grainings? There is; consider the following possibility: Regard linear positivity (or EP) as the fundamental rule determining the probabilities of histories in quantum mechanics. The resulting probabilities satisfy the sum rules exactly as discussed in Section III.B. Medium decoherence then becomes an approximate notion, useful in characterizing classicality, giving through (3.4) an approximation to the fundamental probabilities 6.1). The degree of approximation can be calculated from 3.5. No practical calculation of the probabilities of the quasiclassical realm or its coarse grainings is likely to be affected by adopting this viewpoint. A consequence, however, is that many more sets of coarse-grained alternative histories nothing like the quasiclassical realm are incorporated into the theory beyond the already large number allowed by approximate medium decoherence. A further consequence is that connection between probabilities and the frequencies of even imaginary ensembles of independent subsystems is lost in general as discussed in Section III.D. Precision is achieved at the consequence of extending the complementary descriptions of the world and possibly at the expense of 
any general frequency interpretation of the resulting probabilities.

A more important reason for considering linear positivity or extended probabilities as a fundamental bases for quantum theory lies in their potential for further generalization. The quantum theory of closed systems summarized in Section II may need to be further generalized to incorporate quantum gravity. That is because the framework in Section II relies on a notion of time supplied by a fixed background spacetime geometry. But, in general relativity, spacetime geometry is a dynamical variable that generally will fluctuate and be without a definite value in a quantum theory of gravity. A generalization of the usual quantum framework is required.

Generalized quantum theory [9, 12, 26, 40, 41] provides a natural framework for constructing generalizations of usual quantum theory that incorporates medium decoherence. Generalizations suitable for quantum gravity have been considered by a number of authors (e.g. [9, 42]). But generalizations based upon extended probabilities would provide even greater scope. Indeed a general quantum mechanical theory could be specified just by giving a real valued function for candidate probabilities on the sets of fine-grained histories.

"Cheshire Puss [said Alice]... would you tell me please, which way should I go from here? That depends a good deal on where you want to get to, said the cat".

\section{Acknowledgments}

Conversations with Murray Gell-Mann over a long period of time were helpful. Thanks are due to Todd Brun, Shelly Goldstein, Jonathan Halliwell, Mark Srednicki, and Gary Horowitz for useful discussions. The work was supported in part by the National Science Foundation under grant PHY0070895 .

\section{APPENDIX: EIGENVALUES OF A PRODUCT OF PROJECTIONS}

The Hermitian part of the product of two non-commuting projections has at least one negative eigenvalue. This appendix gives a proof of this undoubtedly well-known fact that the author learned from G.T. Horowitz.

Let $P_{a}$ and $P_{b}$ be the two non-commuting projections and let $G \equiv P_{a} P_{b}+P_{b} P_{a}$ denote their Hermitian product. Let $\lambda_{i}$ and $|i\rangle$ denote the eigenvalues and eigenvectors of $G$. The expectation value in a state $|\Psi\rangle$ is

$$
\langle\Psi|G| \Psi\rangle=\sum_{i} \lambda_{i}|\langle i \mid \Psi\rangle|^{2}
$$

If there is one vector $|\Psi\rangle$ for which $\langle\Psi|G| \Psi\rangle$ is negative then $G$ must have one negative eigenvalue since otherwise (A.1) is positive. We now construct such a vector.

Any vector $|\Psi\rangle$ can be divided into orthogonal vectors $\left|\Psi_{1}\right\rangle$ and $\left|\Psi_{0}\right\rangle$ that lie in the subspace $P_{a}$ and the subspace orthogonal to it, viz.

$$
|\Psi\rangle=\left|\Psi_{1}\right\rangle+\left|\Psi_{0}\right\rangle, \quad P_{a}\left|\Psi_{0}\right\rangle=0, \quad P_{a}\left|\Psi_{1}\right\rangle=\left|\Psi_{1}\right\rangle
$$

We now show how to pick $\left|\Psi_{0}\right\rangle$ and $\left|\Psi_{1}\right\rangle$ to make $\langle\Psi|G| \Psi\rangle$ negative.

With the decomposition A.2

$$
\langle\Psi|G| \Psi\rangle=2\left[\left\langle\Psi_{1}\left|P_{b}\right| \Psi_{1}\right\rangle+\operatorname{Re}\left\langle\Psi_{1}\left|P_{b}\right| \Psi_{0}\right\rangle\right]
$$

For given $\left|\Psi_{1}\right\rangle, P_{b}\left|\Psi_{1}\right\rangle$ cannot be orthogonal to every vector in $P_{a}$, or $P_{b}$ would commute with $P_{a}$ contrary to assumption. Therefore pick $\left|\Psi_{0}\right\rangle$ so that $\operatorname{Re}\left\langle\Psi_{1}\left|P_{b}\right| \Psi_{0}\right\rangle$ is nonvanishing. If $\operatorname{Re}\left\langle\Psi_{1}\left|P_{b}\right| \Psi_{0}\right\rangle>0$ replace $\left|\Psi_{0}\right\rangle$ by $-\left|\Psi_{0}\right\rangle$ so that $\operatorname{Re}\left\langle\Psi_{1}\left|P_{b}\right| \Psi_{0}\right\rangle<0$. Now make $\left|\Psi_{0}\right\rangle$ large enough that the negative second term in A.3 is larger than the positive first term. The result is a $|\Psi\rangle$ such that $\langle\Psi|G| \Psi\rangle \leq 0$.

The case of two one-dimensional projections

$$
P_{a}=|a\rangle\left\langle a\left|\quad P_{b}=\right| b\right\rangle\langle b|
$$

but with $\langle a \mid b\rangle \neq 0$ gives a concrete illustration of the above result. The the eigenvectors of $G$ lie in the two-dimensional space spanned by $|a\rangle$ and $|b\rangle$ and are easily calculated. The two eigenvalues are

$$
\lambda_{ \pm}=c^{2} \pm c, \text { where } c \equiv|\langle a \mid b\rangle| \text {. }
$$

Since $c \leq 1, \lambda_{-}$is evidently negative unless $c=0$ or $c=1$. In either of these cases the projectors commute.
[1] R.B. Griffiths, Consistent Quantum Theory, Cambridge University Press, Cambridge (2002).

[2] R. Omnès, Interpretation of Quantum Mechanics, Princeton University Press, Princeton (1994).

[3] M. Gell-Mann, The Quark and the Jaguar, W. Freeman, San Francisco (1994)

[4] M. Gell-Mann and J.B. Hartle, Alternative Decohering Histories in Quantum Mechanics, in the Proceedings of the 25th International Conference on High Energy Physics, Singapore, August, 2-8, 1990, ed. by K.K. Phua and Y. Yamaguchi, South East Asia Theoretical Physics Association and Physical Society of Japan, distributed by World Scientific, Singapore (1990).
[5] S. Goldstein and D. Page, Linearly Positive Histories - Probabilities for a Robust Family of Sequences of Quantum Events, Phys. Rev. Lett. 74, 3715 (1995); gr-qc/9403055

[6] For an introductory review in the present notation, see J.B. Hartle, The Quantum Mechanics of Closed Systems, in Directions in General Relativity, Volume 1: A Symposium and Collection of Essays in honor of Professor Charles W. Misner's 60th Birthday, ed. by B.-L. Hu, M.P. Ryan, and C.V. Vishveshwara, Cambridge University Press, Cambridge (1993); gr-qc/9210006

[7] L. Diosi, Critique of Weakly Decohering and Linearly Positive Histories, unpublished; gr-qc/9409017

[8] J.B. Hartle, R. Laflamme, and D. Marolf, Conservation Laws 
in the Quantum Mechanics of Closed Systems, Phys. Rev. D 51, 7007 (1995); gr-qc/9410006

[9] J.B. Hartle, Spacetime Quantum Mechanics and the Quantum Mechanics of Spacetime in Gravitation and Quantizations, Proceedings of the 1992 Les Houches Summer School, edited by B. Julia and J. Zinn-Justin, Les Houches Summer School Proceedings Vol. LVII, North Holland, Amsterdam (1995); gr-qc/9304006 A précis of these lectures is given in J.B. Hartle, Quantum Mechanics at the Planck Scale, talk given at the Workshop on Physics at the Planck Scale, Puri, India, December 1994; gr-qc/9508023

[10] D. Finkelstein, Trans. N.Y. Acad. Sci. 25, 621 (1963); J.B. Hartle, Quantum Mechanics of Individual Systems, Am. J. Phys. 36, 704 (1968); R.N. Graham, in The Many-Worlds Interpretation of Quantum Mechanics, ed. by B. DeWitt and R.N. Graham, Princeton University Press, Princeton (1973); E. Farhi, J. Goldstone, and S. Gutmann, Annals of Phys. (N.Y.) 192, 368 (1989); Y. Ohkuwa, Decoherence Functional and Probability Interpretation, Phys. Rev. D 48, 1781 (1993); S. Coleman and A. Lesniewski, unpublished; S. Gutmann, Using Classical Probability to Guarantee Probabilities of Infinite Quantum Sequences, Phys. Rev. A 52, 3560 (1995); quant-ph/9506016

[11] M. Gell-Mann and J.B. Hartle, Time Symmetry and Asymmetry in Quantum Mechanics and Quantum Cosmology, in Proceedings of the NATO Workshop on the Physical Origins of Time Asymmetry, Mazagón, Spain, September 30-October4, 1991 ed. by J. Halliwell, J. Pérez-Mercader, and W. Zurek, Cambridge University Press, Cambridge (1993); gr-qc/9309012

[12] M. Gell-Mann and J.B. Hartle, Quantum Mechanics in the Light of Quantum Cosmology, in Complexity, Entropy, and the Physics of Information, SFI Studies in the Sciences of Complexity, Vol. VIII, ed. by W. Zurek, Addison Wesley, Reading, MA (1990).

[13] J.B. Hartle, Quasiclassical Domains In A Quantum Universe, in Proceedings of the Cornelius Lanczos International Centenary Conference, North Carolina State University, December 1992, ed. by J.D. Brown, M.T. Chu, D.C. Ellison, R.J. Plemmons, SIAM, Philadelphia, (1994); gr-qc/9404017

[14] M. Gell-Mann and J.B. Hartle, Classical Equations for Quantum Systems, Phys. Rev. D 47, 3345 (1993); gr-qc/9210010

[15] M. Gell-Mann and J.B. Hartle, unpublished.

[16] R. Landauer, IBM J. Res. Develop. 3, 183 (1961).

[17] R.P. Feynman and A. Hibbs, Quantum Mechanics and Path Integrals, McGraw-Hill, New York (1965).

[18] H.F. Dowker and J. Halliwell, Phys. Rev. D 46, 1580 (1992).

[19] Y. Aharonov and L. Vaidman, Complete Description of a Quantum System at a Given Time, J. Phys. A 24 2315, (1991).

[20] A. Kent, Consistent Sets Yield Contrary Influences in Quantum Theory, Phys. Rev. Lett. 78, 2874 (1997); gr-qc/9604012

[21] R.B. Griffiths and J.B. Hartle, Comment on Consistent Sets Yield Contrary Inferences in Quantum Theory, Phys. Rev. Lett. 81, 1981 (1998); gr-qc/9710025

[22] J.B. Hartle, Spacetime Coarse Grainings in Non-Relativistic Quantum Mechanics, Phys. Rev. D 44, 3173 (1991).

[23] N. Yamada and S. Takagi, Prog. Theor. Phys. 85, 985 (1991); ibid., Prog. Theor. Phys. 86, 599 (1991); ibid., Prog. Theor. Phys. 87, 77 (1992).

[24] C. Caves, Quantum Mechanics of Measurements Distributed in Time: A Path Integral Formulation, Phys. Rev.D 33, 1643
(1986).

[25] R.J. Micanek and J.B. Hartle, Nearly Instantaneous Alternatives in Quantum Mechanics, Phys. Rev. A 54, 3795-3800 (1996); quant-ph/9602023

[26] J.B. Hartle, The Quantum Mechanics of Cosmology, in Quantum Cosmology and Baby Universes: Proceedings of the 1989 Jerusalem Winter School for Theoretical Physics, ed. by S. Coleman, J.B. Hartle, T. Piran, and S. Weinberg, World Scientific, Singapore (1991) pp. 65-157, Section II.10.

[27] J.B. Hartle, Quantum Pasts and the Utility of History, Physica Scripta T76, 67-77 (1998); gr-qc/9712001

[28] R.P. Feynman, Negative Probability in Quantum Implications: Essays in Honor of David Bohm, ed. by B.J. Hiley and F.D. Peat, Routledge and Kegan Paul, London (1987).

[29] References [2, 13, 14, 30, 31, 32, 33] illustrate the diversity of approaches to defining classicality. They are generally not the earliest or the latest.

[30] M. Gell-Mann and J.B. Hartle, Strong Decoherence, in the Proceedings of the 4th Drexel Symposium on Quantum Non-Integrability - The Quantum-Classical Correspondence, Drexel University, September 8-11, 1994, ed. by D.H. Feng and B.-L. Hu, International Press, Boston/Hong-Kong; gr-qc/9509054

[31] C. Anastopoulos, Information Measures and Classicality in Quantum Mechanics, Phys. Rev. D 59, 045001 (1999); quant-ph/9805005

[32] W. Zurek, Decoherence, Einselection and the Quantum Origin of the Classical, Rev. Mod. Phys. 75, 75 (2003).

[33] T. Brun and J.B. Hartle, Classical Dynamics of the Quantum Harmonic Chain, Phys. Rev. D 60, 123503 (1999); quant-ph/9905079

[34] E. Joos and H.D. Zeh, Zeit. Phys. B59, 223 (1985).

[35] A. Caldeira and A. Leggett, Path Integral Approach to Quantum Brownian Motion, Physica 121A, 587 (1983).

[36] W. Zurek, in Non-Equilibrium Quantum Statistical Physics, ed. by G. Moore and M. Scully, Plenum Press, New York (1984); quant-ph/0302044

[37] J. Halliwell, Somewhere in the Universe: Where is the Information Stored When Histories Decohere, Phys. Rev. D 60, 105031 (1999); quant-ph/9902008

[38] J. Halliwell, Decoherence of Histories and Hydrodynamic Equations for Linear Oscillator Chain (2003); quant-ph/0305084

[39] H.F. Dowker and A. Kent, On the Consistent Histories Approach to Quantum Mechanics, J. Stat. Phys. 82, 1574 (1996); gr-qc/9412067

[40] C.J. Isham, Quantum Logic and the Histories Approach to Quantum Theory, J. Math. Phys. 35, 2157 (1994); quant-ph/9308006

[41] C.J. Isham and N. Linden, Quantum Temporal Logic and Decoherence Functionals in the Histories Approach to Generalized Quantum Theory, J. Math. Phys., 35, 5452 (1994); gr-qc/9405029

[42] K. Savvidou, General Relativity Histories Theory I: The Spacetime Character of the Canonical Description; gr-qc/030634. General Relativity Histories Theory II: Invariance Groups; gr-qc/0306036 